Article

\title{
Chromene- and Quinoline-3-Carbaldehydes: Useful Intermediates in the Synthesis of Heterocyclic Scaffolds
}

\author{
Djenisa H. A. Rocha ${ }^{1,2}{ }^{1}$, Vasco F. Batista ${ }^{1}$, Emanuel J. F. Balsa ${ }^{1}\left({ }^{1}\right.$, Diana C. G. A. Pinto ${ }^{1, *(\mathbb{D}}$ \\ and Artur M. S. Silva ${ }^{1}$ (D) \\ 1 LAQV-REQUIMTE \& Department of Chemistry, Campus de Santiago, University of Aveiro, 3810-193 Aveiro, \\ Portugal; djenisa@ua.pt (D.H.A.R.); vfb@ua.pt (V.F.B.); emanuelbalsa@ua.pt (E.J.F.B.); \\ artur.silva@ua.pt (A.M.S.S.) \\ 2 CICECO-Aveiro Institute of Materials \& Department of Chemistry, Campus de Santiago, \\ University of Aveiro, 3810-193 Aveiro, Portugal \\ * Correspondence: diana@ua.pt
}

Academic Editor: Gianfranco Favi

Received: 27 July 2020; Accepted: 19 August 2020; Published: 20 August 2020

\begin{abstract}
Chromenes and quinolines are recognized as important scaffolds in medicinal chemistry. Herein, the efficient use of chromene- and quinoline-3-carbaldehydes to synthesize other valuable heterocycles is described. These carbaldehydes are obtained in excellent yields through the Vilsmeyer-Haack reaction of flavanones and azaflavanones. Protocols towards the synthesis of new heterocycles, such as $3 H$-chromeno[3-clquinolines, (Z/E)-2-aryl-4-chloro-3-styryl-2H-chromenes, and (E)-2-aryl-4-chloro-3-styrylquinoline-1(2H)-carbaldehydes were established. Altogether, we demonstrate the value of chromene- and quinoline-3-carbaldehydes as building blocks.
\end{abstract}

Keywords: chromene-3-carbaldehydes; 3-styryl-2H-chromenes; quinoline-3-carbaldehydes; $3 H$-chromeno [3,4-c]quinolines; 3-styrylquinoline-1(2H)-carbaldehydes; Wittig reaction

\section{Introduction}

The synthesis of new heterocyclic compounds is a significant aspect of medicinal chemistry due to the recognized value of this type of compound in the development of new drugs. In this regard, quinoline and chromene cores are interesting frameworks owing to their established medicinal value. Biological activities, such as anticancer [1,2], antimicrobial [3-5], anti-Alzheimer's [6], antioxidant [7], and cardiovascular [8], can be highlighted. These nuclei can be found in natural compounds [9-12] and synthetic materials [13-15].

In this manuscript, the usefulness of 4-chloro(chromene- and quinoline)-3-carbaldehydes in the synthesis of other significant heterocycles is described. Moreover, the 4-chloro(chromeneand quinoline)-3-carbaldehydes 2 are easily obtained, in good to excellent yields, through a Vilsmeyer-Haack reaction (Scheme 1) using flavanones $\mathbf{1}(\mathrm{X}=\mathrm{O})$ and 2-aryl-2,3-dihydroquinolines $\mathbf{1}$ ( $\mathrm{X}=\mathrm{NH}$, azaflavanones), respectively. Recently we established a straightforward protocol to achieve, in excellent yields, these flavanone and azaflavanone derivatives [16].

Among the significant heterocycles described are indole and quinoline derivatives recognized bioactive alkaloids, for which-New synthetic methodologies are always essential to be established. Moreover, the study of the use of 4-chloro(chromene- and quinoline)-3-carbaldehydes 2 in Wittig reactions is also described and allowed the synthesis of interesting derivatives bearing a 3-styryl moiety. The work herein reported, besides the diversity of structures obtained, also demonstrates the scope of the transformations. 


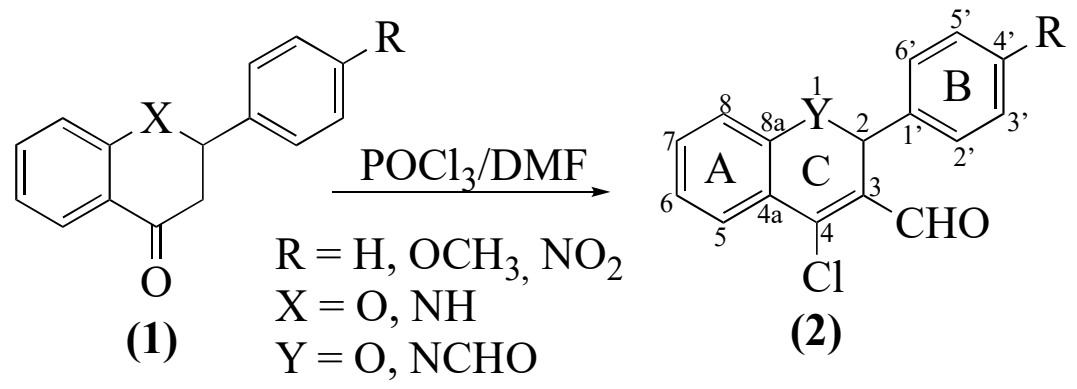

Scheme 1. Synthesis of 4-chlorochromene-3-carbaldehydes 2a-c and 4-chloroquinoline-3-carbaldehydes $\mathbf{2 d - f .}$

\section{Results and Discussion}

The Vilsmeyer-Haack formylation is an everyday procedure in our group $[17,18]$ that was easily used in the formylation of flavanones $\mathbf{1}$ (Scheme $1, X=O$ ) and, in the case of azaflavanones $\mathbf{1}$, (Scheme 1 , $\mathrm{X}=\mathrm{NH}$ ), required a few optimization steps. The quantities used and the reaction time were adjusted to ensure the total formylation at bot $\mathrm{H}-\mathrm{N}-1$ and $\mathrm{C}-3$ and consequently avoid monoformylated derivatives.

Nuclear magnetic resonance experiments confirmed the structures of both 2-aryl-4-chloro2H-chromene-3-carbaldehydes $\mathbf{2 a - c}$ and 2-aryl-4-chloro-1,2-dihydroquinoline-1,3-dicarbaldehydes $\mathbf{2} \mathbf{d}-\mathbf{f}$. The most typical features in their ${ }^{1} \mathrm{H}-\mathrm{NMR}$ spectra are the singlets at $\delta=6.33-6.47$ and $6.88-7.00 \mathrm{ppm}$ corresponding to the resonance of $\mathrm{H}-2$, respectively, for compounds $\mathbf{2 a}-\mathbf{c}$ and $\mathbf{2 d}-\mathbf{f}$. Other important signals are seen at $\delta=10.27-10.32$ and $10.30-10.33 \mathrm{ppm}$ due to the formyl protons' resonances. In the ${ }^{13} \mathrm{C}-\mathrm{NMR}$ spectra, the signal due to the $3-\mathrm{CHO}$ at $\delta=187.6-188.2 \mathrm{ppm}$ and the signal due to C-2 at $\delta=73.8-75.0$ ppm for compounds $2 \mathbf{a}-\mathbf{c}$ and at $\delta=50.2-50.6 \mathrm{ppm}$ for compounds $\mathbf{2} \mathbf{d}-\mathbf{f}$ should be highlighted.

The indole nucleus is a prominent constituent of alkaloids such as serotonin and melatonin $[19,20]$ and is also present in many bioactive alkaloids. Antitumoral [21,22], anti-arrhythmic [19], anti-inflammatory [23], antimicrobial [24], and antihypertensive properties are amongst the reported biological activities. The synthesis of indole derivatives is largely explored due to relevant biological applications described for this scaffold, and there are reports on the synthesis of indole hybrids [25-27]. We thought it would be interesting to obtain chromene-indole hybrids, so we studied the reaction of 2-aryl-4-chloro- $2 H$-chromene-3-carbaldehydes $2 \mathbf{a}-\mathbf{c}$ with indole (3). Unexpectedly, complex mixtures of compounds were obtained. The two major products' isolation suggested that they were derivatives presenting one and two indole units, respectively.

Furthermore, the 2-aryl-4-chloro-2H-chromene-3-carbaldehyde derivative was recovered as well as some degradation products. The amount of DMSO used was optimized to a minimum, to allow an excellent stirring. The best reaction temperature was established at $130^{\circ} \mathrm{C}$, to avoid the loss of the formyl group. The amount of indole 3 added was established to prevent mixtures of hybrids and maximize the yield of the two indole unit hybrid. This careful optimization of the reaction conditions allowed us to obtain a single compound (Scheme 2) in very good yields (60 to $84 \%$ ).

The MS spectrum of the obtained compound suggested that it should contain two indole units. Furthermore, the NMR experiments confirmed this hypothesis and allowed us to establish that the obtained compounds were 3,3'-[(2-aryl-4-chloro-2H-chromen-3-yl)methylene]bis( $1 H$-indoles) $4 \mathbf{a}-\mathbf{c}$ as depicted in Scheme 2. We believe that the indole carbon C-3 nucleophilicity is high and that the formyl carbon electrophilicity favors the reaction with a second indole unit. The main NMR features are: (i) $\mathrm{NH}$ proton resonances at $\delta=10.32-10.61$ and $11.06-11.16 \mathrm{ppm}$, appearing as doublets $(J \approx 2 \mathrm{~Hz})$ due to their coupling with $\mathrm{H}-2$ in each indole unit; (ii) methinic proton of the carbon linked to the two indole units, a singlet at $\delta=6.14-6.25 \mathrm{ppm}$. 


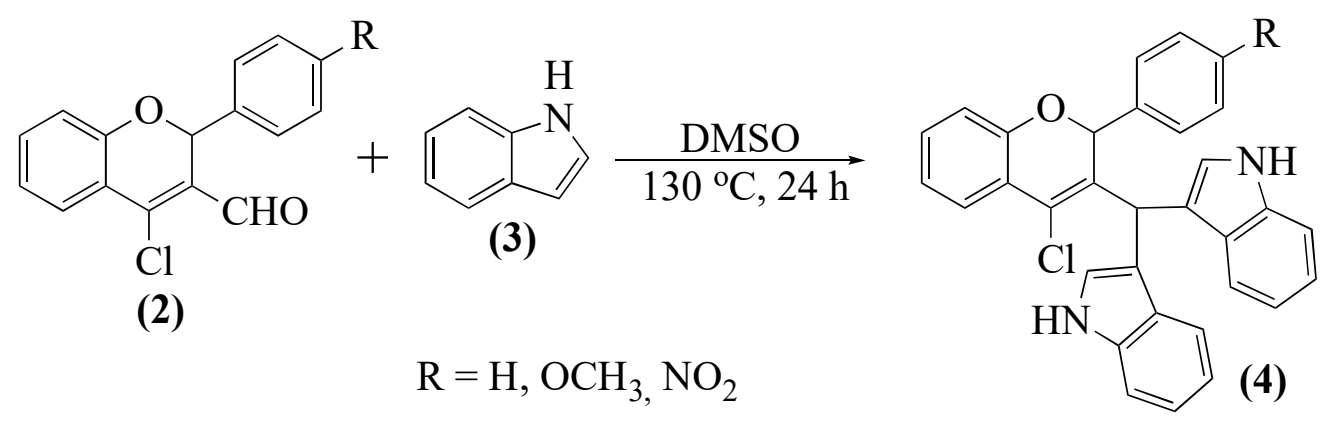

Scheme 2. Synthesis of 3,3'-[(2-aryl-4-chloro-2H-chromen-3-yl)methylene $]$ bis( $1 H$-indoles) $4 \mathbf{a}-\mathbf{c}$.

Next, the reactivity of 2-aryl-4-chloro-2H-chromene-3-carbaldehydes $\mathbf{2 a - c}$ with anilines $\mathbf{5}$ was studied. The main objective was to obtain quinoline derivatives by forming an imine intermediate, followed by its cyclization with the elimination of hydrochloric acid (Scheme 3).

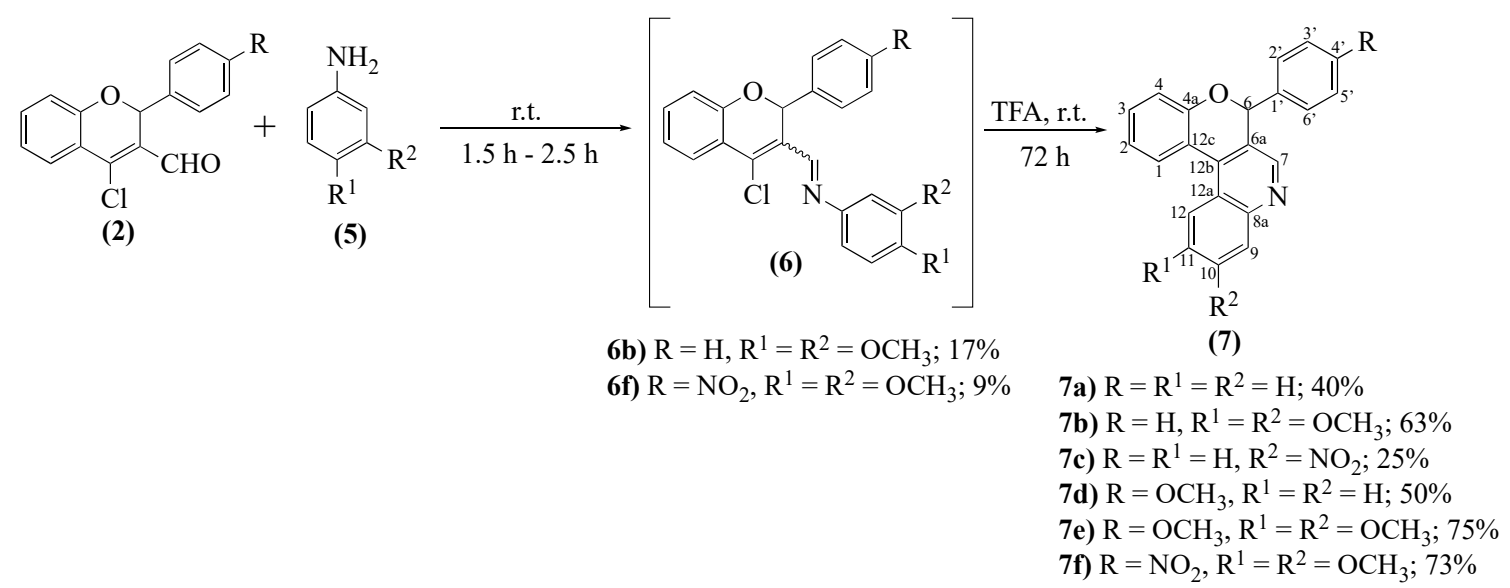

Scheme 3. Synthesis of 6-aryl-6H-chromeno[3,4-c]quinolines 7a-f.

The first attempts, reacting 4-chloro-2-phenyl-2H-chromene-3-carbaldehyde $2 \mathbf{a}$ with aniline, gave a mixture of products and indications that the imine derivative was either not stable, or not able to resist the purification steps. Therefore, we decided to control the reaction by TLC, adding TFA to promote the in situ cyclization immediately after the consumption of the $\mathbf{2 a}$. This procedure proved efficient and allowed the synthesis of the desired 6-phenyl-6H-chromeno[3,4-c]quinoline 7a in moderate yield. Naturally, the reaction scope was further evaluated (Scheme 3). Although the reaction occurs with certain success, we notice that the first step requires variable reaction times (from 1.5 to $2.5 \mathrm{~h}$ ), whereas the second step was accomplished in an additional $72 \mathrm{~h}$. Other relevant results include the lower or nonexistent reactivity of derivatives having nitro groups as substituents; only two novel compounds, $7 \mathbf{c}$ and $\mathbf{7 f}$, were obtained, the former in low yield (25\%). On the other hand, the presence of activating groups in the chromene-3-carbaldehydes, such as $\mathrm{OCH}_{3}$, improve the yield, cases of $\mathbf{7 d}$ $(50 \%)$, and $7 \mathbf{e}(75 \%)$. That is more evident when two methoxy substituents are present in aniline, which increased reactivity, and all derivatives were obtained in very good yields $(7 \mathbf{b}, 63 \% ; 7 \mathbf{e}, 75 \%, 7 \mathrm{f}, 73 \%)$. We were also able to isolate two imine derivatives (Scheme 3 ) in low yields, and $\mathbf{6 f}$ was obtained only when the second step was shortened $48 \mathrm{~h}$.

The main features in the NMR characterization of 6-aryl-6H-chromeno[3,4-c]quinolines $7 \mathbf{a}-\mathbf{f}$ are the two singlets at $\delta=6.32-6.44$ and 7.30-7.60 ppm due to, respectively, H-6 and H-7 resonances. Another typical signal is the double doublet appearing at $\delta=8.40-8.55 \mathrm{ppm}$ and corresponding to the $\mathrm{H}-1$ resonance. These signals were not only in agreement with the proposed structure (Scheme 3) but also showed essential connectivities in the HMBC spectra that allowed the full characterization of the synthesized 6-aryl-6H-chromeno[3,4-c]quinolines 7a-f. 
3-Styrylchromenes constitute a small group of synthetic compounds recently reported for their antiviral [3], cytotoxic [28], and antimicrotubular activity [29]. Several methods for the synthesis of the 3-styryl-2H-chromene nucleus have been described, including the Horner-Wadsworth-Emmons [3] and Wittig [29] reactions. Considering this, we decided to assess the reactivity of 2-aryl-4-chloro$2 \mathrm{H}$-chromene-3-carbaldehydes $\mathbf{2 a - c}$ in a Wittig reaction with aromatic ylides 9 (Scheme 4). Our first approach was to use a previously established procedure [30], although with some adaptations, mainly in the reaction times and the amount of base. The two isomers were always obtained, with a clear preference for (Z)-2-aryl-4-chloro-3-styryl-2H-chromenes 11a-f (Table 1, Scheme 4), as predicted by Wittig reaction selectivity [31].

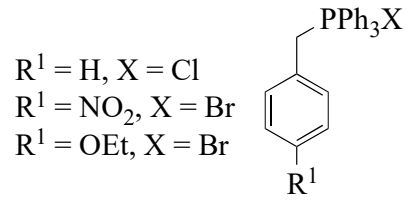

(8)

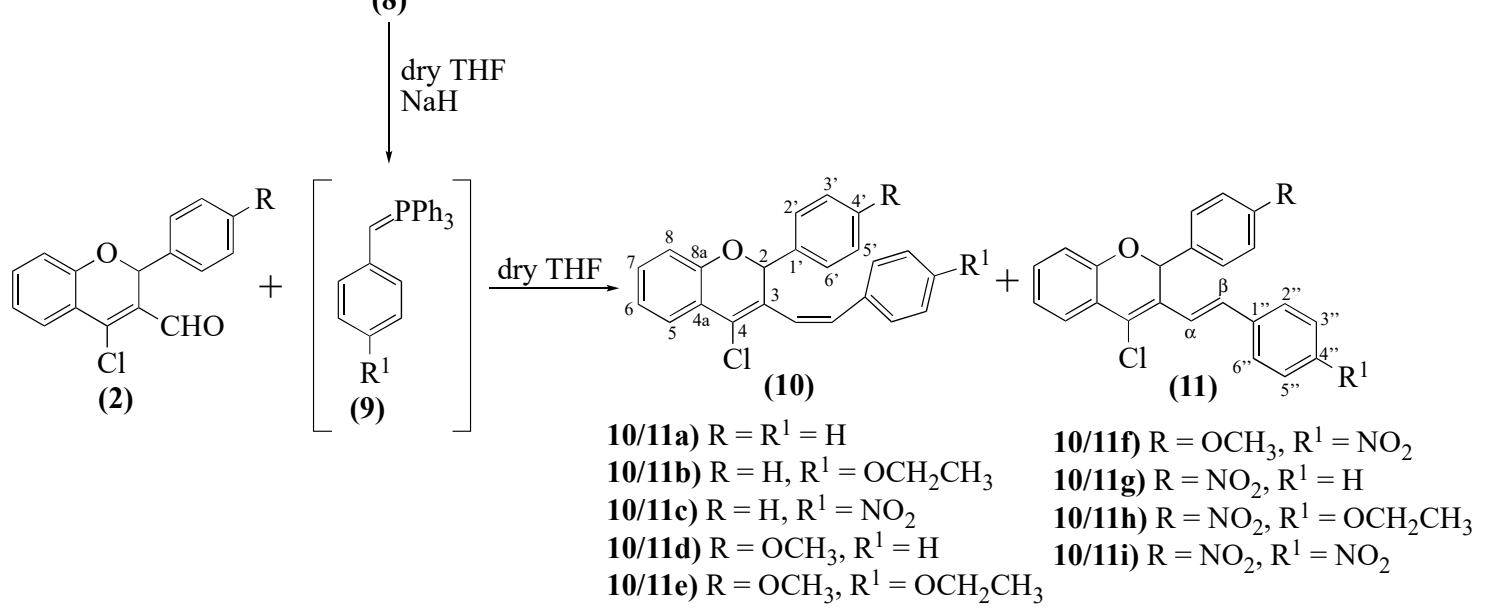

Scheme 4. Synthesis of (Z- and E)-2-aryl-4-chloro-3-styryl-2H-chromenes 10a-i and 11a-i.

The optimization steps demonstrated that the Wittig reaction results are dependent on both the aldehyde $\mathbf{2}$ and the ylide $\mathbf{9}$. It is clear that the ylide $\mathbf{9 c}$, having an electron-donating group, requires less time to be formed, depends on the amount of base used and, in our opinion, also on the solvent dryness; freshly distilled THF holds better results (Table 1). The aldehyde 2a was less reactive. It requires longer reaction times, and the major isomer is obtained in lower yields (Table 1). Another interesting result is that aldehyde $2 \mathrm{c}$ presents better results if the second step is performed at room temperature (Table 1); in fact, reflux promotes aldehyde decomposition instead of improving its reactivity.

The most important features of the ${ }^{1} \mathrm{H}-\mathrm{NMR}$ spectra of 2-aryl-4-chloro-3-styryl-2 $\mathrm{H}$-chromenes 10a-i and 11a-i are the signals of the vinylic protons, which allowed the establishment of the system absolute configuration. In the (Z)-isomer 10a-i, the resonance of $\mathrm{H}-\beta$ (6.56-6.74 ppm) appears at higher frequency values than those of $\mathrm{H}-\alpha(6.22-6.63 \mathrm{ppm})$, and the stereochemistry of the vinylic system was established as $(Z)$ due to typical coupling constant values $\left({ }^{3} J_{\mathrm{H}-\alpha-\mathrm{H}-\beta} 12.1-12.3 \mathrm{~Hz}\right)$. In contrast, in the $(E)$-isomer 11a-i, is the resonance of $\mathrm{H}-\alpha(7.42-7.75 \mathrm{ppm})$ that appears at higher frequency values, due to the chlorine atom's proximity, than those of H- $\beta$ (6.42-6.50 ppm). The vinylic system's stereochemistry was established as $(E)$ due to typical coupling constant values $\left({ }^{3} J_{\mathrm{H}-\alpha-\mathrm{H}-\beta} 16.5-16.6 \mathrm{~Hz}\right)$ and confirmed by the NOESY experiments. 
Table 1. Optimization of reaction conditions for the synthesis of ( $\mathrm{Z}$ and $E)$-2-aryl-4-chloro-3-styryl-2Hchromenes 10a-f and 11a-i.

\begin{tabular}{|c|c|c|c|c|c|c|c|}
\hline Entry & Comp. 2 & Ylide & $\begin{array}{l}\mathrm{NaH}^{\mathrm{a}} \\
\text { (Equiv) }\end{array}$ & $\begin{array}{c}\text { 1st Step } \\
\text { (Time) }\end{array}$ & $\begin{array}{l}\text { 2nd Step } \\
\text { (Time) }\end{array}$ & $\begin{array}{l}\text { (Z)-isomer } \\
\text { (Yield, \%) }\end{array}$ & $\begin{array}{l}(E) \text {-isomer }{ }^{d} \\
(\text { Yield, \%) }\end{array}$ \\
\hline 1 & \multirow{6}{*}{$2 \mathrm{a}(\mathrm{R}=\mathrm{H})$} & \multirow{4}{*}{$9 a$} & 5 & $3 \mathrm{~h}$ & $3 \mathrm{~h}$ & 10a, 18 & $11 a, 10$ \\
\hline 2 & & & 7 & $3 \mathrm{~h}$ & $3 \mathrm{~h} 30 \mathrm{~min}$ & $10 a, 25$ & 11a, 19 \\
\hline 3 & & & 7 & $1.5 \mathrm{~h}$ & $21 \mathrm{~h}$ & $10 a, 32$ & $11 a, 5$ \\
\hline 4 & & & 7 & $20 \mathrm{~min}$ & $24 \mathrm{~h}$ & $10 a, 32$ & $11 a, 25$ \\
\hline 5 & & $9 b$ & 7 & $10 \mathrm{~min}$ & $3 \mathrm{~h} 45 \mathrm{~min}$ & 10b, 45 & $11 b, 25$ \\
\hline 6 & & $9 c$ & 7 & $3 \mathrm{~min}$ & $24 \mathrm{~h}$ & $10 c, 41$ & 11c, 33 \\
\hline 7 & \multirow{3}{*}{$\mathbf{2 b}\left(\mathrm{R}=\mathrm{OCH}_{3}\right)$} & $9 a$ & 7 & $15 \mathrm{~min}$ & $3 \mathrm{~h} 50 \mathrm{~min}$ & 10d, 50 & 11d, 28 \\
\hline 8 & & $9 b$ & 8 & $5 \mathrm{~min}$ & $3 \mathrm{~h} 30 \mathrm{~min}$ & $10 \mathbf{e}, 50$ & $11 \mathrm{e}, 20$ \\
\hline 9 & & $9 \mathrm{c}$ & 8 & $3 \mathrm{~min}$ & $3 \mathrm{~h}$ & $10 f, 53$ & 11f, 35 \\
\hline 10 & \multirow{8}{*}{$2 \mathrm{c}\left(\mathrm{R}=\mathrm{NO}_{2}\right)$} & \multirow{4}{*}{ 9a } & 8 & $15 \mathrm{~min}$ & $5 \mathrm{~h}$ & $10 g_{,}-$ & $11 g,-$ \\
\hline 11 & & & 8 & $15 \mathrm{~min}$ & $3 \mathrm{~h}$ & $10 g_{\prime}-$ & $11 \mathrm{~g},-$ \\
\hline 12 & & & 9 & $10 \mathrm{~min}$ & $24 h^{f}$ & $10 \mathrm{~g}, 27$ & $11 \mathrm{~g},-$ \\
\hline 13 & & & 3 & $10 \mathrm{~min}$ & $40 \min ^{f}$ & $10 \mathrm{~g}, 34$ & 11g, 15 \\
\hline 14 & & \multirow{2}{*}{$9 b$} & 7 & $2 \mathrm{~min}$ & $4 \mathrm{~h}$ & 10h, 20 & 11h, - \\
\hline 15 & & & 7 & $3 \mathrm{~min}$ & $1 \mathrm{~h}^{\mathrm{f}}$ & 10h, 45 & $11 h, 24$ \\
\hline 16 & & \multirow{2}{*}{$9 c$} & 8 & $6 \mathrm{~min}$ & $4 \mathrm{~h}$ & $10 \mathrm{i},-$ & $11 i,-$ \\
\hline 17 & & & 3 & $10 \min ^{e}$ & $2 h^{f}$ & $10 \mathrm{i}, 52$ & $11 i, 27$ \\
\hline
\end{tabular}

${ }^{a}$ Molar excess; ${ }^{b}$ Time to obtain the ylide; ${ }^{c}$ Time after the aldehyde addition; ${ }^{\mathrm{d}}$ Isolated compound yield in $\% ;{ }^{\mathrm{e}}$ THF freshly dried; ${ }^{f}$ Second step at room temperature.

The success achieved in the synthesis of 2-aryl-4-chloro-3-styryl-2H-chromene derivatives prompt us to use the 2-aryl-4-chloro-1,2-dihydroquinoline-1,3-dicarbaldehydes $\mathbf{2 d - f}$, aiming to obtain new (Z- and E)-2-aryl-4-chloro-3-styrylquinolines. Therefore, we considered a previously established procedure for the synthesis of 3-styryl-4-quinolones [32], using the reaction of

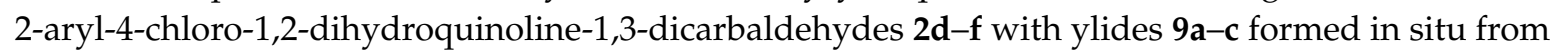
the phosphonium salts $\mathbf{8 a - c}$ (Scheme 5). A color change and TLC analysis controlled the formation of ylide, being the ideal time for the ylides formation $15 \mathrm{~min}, 3 \mathrm{~min}$, and $7 \mathrm{~min}$, respectively, for $9 \mathrm{a}$, $\mathbf{9 b}$, and $\mathbf{9 c}$. After the ylide formation, aldehydes $\mathbf{2 d}-\mathbf{f}$ were added to the reaction mixture. The first results were not as good as expected (Table 2; Entries 1, 8, and 15). As so, the reaction conditions were individually studied (Table 2). The analysis of Table 2 shows that the results depend on the aldehyde's reactivity and that the second step's reaction time is a crucial parameter for the transformation.

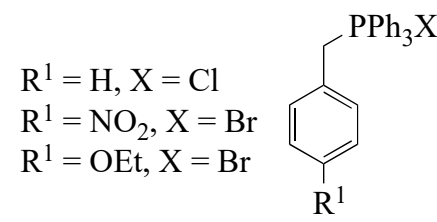

(8) dry THF $\mathrm{NaH}$<smiles>[R]c1ccc(C=[Pb])cc1</smiles>

$$
\begin{aligned}
& \text { 12a) } \mathrm{R}=\mathrm{R}^{1}=\mathrm{H} \\
& \text { 12c) } \mathrm{R}=\mathrm{H}, \mathrm{R}^{1}=\mathrm{NO}_{2} \\
& \text { 12d) } \mathrm{R}=\mathrm{OCH}_{3}, \mathrm{R}^{1}=\mathrm{H} \\
& \text { 12e) } \mathrm{R}=\mathrm{OCH}_{3}, \mathrm{R}^{1}=\mathrm{OCH}_{2} \mathrm{CH}_{3} \\
& \text { 12f) } \mathrm{R}=\mathrm{OCH}_{3}, \mathrm{R}^{1}=\mathrm{NO}_{2} \\
& \text { 12g) } \mathrm{R}=\mathrm{NO}_{2}, \mathrm{R}^{1}=\mathrm{H} \\
& \text { 12h) } \mathrm{R}=\mathrm{NO}_{2}, \mathrm{R}^{1}=\mathrm{OCH}_{2} \mathrm{CH}_{3}
\end{aligned}
$$<smiles>[R]c1ccc(C2C(C=O)=C(Cl)c3ccccc3N2C=O)cc1</smiles>

(2)

(9)

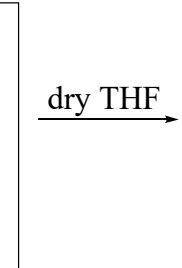<smiles>[R]c1ccc(C=CC2=C(Cl)c3ccccc3N(C=O)C2c2ccc([R])cc2)cc1</smiles><smiles>[R]c1ccc(/C=C\C2=C(Cl)c3ccccc3N(C=O)C2c2ccc([R])cc2)cc1</smiles>

(13)

Scheme 5. Synthesis of (Z- and E)-2-aryl-4-chloro-3-styrylquinoline-1(2H)-carbaldehydes 12a-i and 13a-i. 
Table 2. Optimization of reaction conditions for the synthesis of (E)-2-aryl-4-chloro-3-styrylquinoline$1(2 H)$-carbaldehydes $\mathbf{1 2 a}-\mathbf{i}$.

\begin{tabular}{|c|c|c|c|c|c|}
\hline Entry & Comp. 2 & Ylide & $\begin{array}{c}\text { 2nd step } \\
\text { (Time) }\end{array}$ & Comp. 12 & $\begin{array}{l}\text { (E)-Isomer b } \\
\text { (Yield, \%) }\end{array}$ \\
\hline 1 & \multirow{7}{*}{$2 d(\mathrm{R}=\mathrm{H})$} & \multirow{3}{*}{$9 a$} & $50 \mathrm{~min}$ & $12 a$ & 35 \\
\hline 2 & & & $1 \mathrm{~h} 20 \mathrm{~min}$ & $12 a$ & 40 \\
\hline 3 & & & $2 \mathrm{~h}$ & $12 a$ & 44 \\
\hline 4 & & \multirow{2}{*}{$9 b$} & $46 \mathrm{~min}$ & $12 b$ & $17^{\mathrm{c}}$ \\
\hline 5 & & & $1 \mathrm{~h} 30 \mathrm{~min}$ & $12 b$ & $35^{c}$ \\
\hline 6 & & \multirow[b]{2}{*}{$9 c$} & $2 \mathrm{~h}$ & $12 \mathrm{c}$ & 33 \\
\hline 7 & & & $3 \mathrm{~h}$ & $12 \mathrm{c}$ & 40 \\
\hline 8 & \multirow{7}{*}{$\mathbf{2 e}\left(\mathrm{R}=\mathrm{OCH}_{3}\right)$} & \multirow{3}{*}{$9 a$} & $45 \mathrm{~min}$ & $12 d$ & 46 \\
\hline 9 & & & $1 \mathrm{~h} 20 \mathrm{~min}$ & $12 d$ & 35 \\
\hline 10 & & & $2 \mathrm{~h} 20 \mathrm{~min}$ & $12 d$ & 20 \\
\hline 11 & & \multirow{2}{*}{$9 b$} & $40 \mathrm{~min}$ & $12 \mathrm{e}$ & 27 \\
\hline 12 & & & $1 \mathrm{~h} 20 \mathrm{~min}$ & $12 e$ & 25 \\
\hline 13 & & \multirow{2}{*}{$9 c$} & $2 \mathrm{~h}$ & $12 \mathrm{f}$ & 43 \\
\hline 14 & & & $3 \mathrm{~h}$ & $12 f$ & 53 \\
\hline 15 & \multirow{5}{*}{$2 \mathrm{f}\left(\mathrm{R}=\mathrm{NO}_{2}\right)$} & \multirow{2}{*}{$9 a$} & $58 \mathrm{~min}$ & $12 \mathrm{~g}$ & 18 \\
\hline 16 & & & $2 \mathrm{~h} 20 \mathrm{~min}$ & $12 \mathrm{~g}$ & 30 \\
\hline 17 & & $9 b$ & $45 \mathrm{~min}$ & $12 \mathrm{~h}$ & 24 \\
\hline 18 & & \multirow{2}{*}{$9 c$} & $55 \mathrm{~min}$ & $12 \mathrm{i}$ & - \\
\hline 19 & & & $2 \mathrm{~h}$ & $12 \mathrm{i}$ & $27^{\mathrm{c}}$ \\
\hline
\end{tabular}

Another significant observation is that in this reaction, the $(E)$-isomer was the major product. In contrast, the $(Z)$-isomer was obtained in vestigial amounts or just detected as an impurity in the ${ }^{1} \mathrm{H}-\mathrm{NMR}$ spectra. For this reason, none of the $(Z)$-isomers were obtained as pure compounds. In a few cases (Table 2; Entries 4, 5, 18, and 19), we could not isolate the (E)-isomer, because they are light-sensitive, especially when in solution or silica gel. In other cases, increasing the second step reaction time allowed the yield improvement (Table 2; Entries 3, 7, 14, and 16).

The ylide's reactivity seems to influence the result, with the ylide $9 \mathrm{c}\left(\mathrm{R}^{1}=\mathrm{NO}_{2}\right)$ as the less reactive, probably due to the electron-withdrawing group. Although $9 \mathbf{b}\left(\mathrm{R}^{1}=\mathrm{OCH}_{2} \mathrm{CH}_{3}\right)$ appeared to be more reactive, with shorter reaction times in a few examples (Table 2; Entries 11 and 17), the yields were not as good as expected. In terms of the aldehyde's reactivity, 2-aryl-4-chloro-1,2-dihydroquinoline-1,3-dicarbaldehyde $\mathbf{2 e}$ is the most reactive. We were able to obtain all (E)-isomers, some in moderate yields (Table 2; Entries 8 and 14). Three crucial conclusions can be reached from these results:

(i) 2-Aryl-4-chloro-1,2-dihydroquinoline-1,3-dicarbaldehydes $\mathbf{2} \mathbf{d}-\mathbf{f}$ are not as reactive as the 2-aryl-4-chloro-3-styryl-2H-chromenes $\mathbf{2 a - c}$;

(ii) The common Wittig reaction stereoselectivity was not observed, the (E)-isomer was obtained;

(iii) The formyl group at C-1 did not undergo Wittig reaction, naturally because of the amide carbonyl group's lower nucleophilicity.

The main features in the ${ }^{1} \mathrm{H}-\mathrm{NMR}$ spectra of $(E)$-2-aryl-4-chloro-3-styrylquinoline-1(2H)-carbaldehydes 12a-i are the resonances of $H-\alpha$ and $H-\beta$, which appear as two doublets at $\delta=7.40-7.74$ and $6.69-6.81 \mathrm{ppm}$, respectively, and have coupling constants $(J \approx 16 \mathrm{~Hz})$ typical of an $(E)$-configuration. The depicted 3-styryl group conformation (Scheme 5 ) was confirmed by NOESY experiments in whicH-NOE correlations between $H-\beta$ and $H-2, H-2^{\prime}$ were observed.

A DFT analysis on the reaction of benzyl triphenylphosphonium ylide with $2 \mathbf{a}$ was performed to rationalize the abnormal selectivity obtained with the used complex aldehydes, considering the possible 
formation of both ( $E$ and $Z$ )-isomers. Previous reports have shown that the Wittig reaction mechanism witH-Non-stabilized and semi-stabilized ylides proceeds through an early four-centre transition state leading to the irreversible formation of a phosphoxetane intermediate; evidence clearly discards the alternative existence of a betaine species. The reaction then passes through a secondary transition state as the phosphoxetane degenerates to the desired alkene product. It has been established in the literature that this first transition state's nature determines the stereochemistry of the reaction, locking the relative position of the aldehyde and ylide irreversibly [31].

Preliminary studies using the low level of theory B3LYP/STO-3G* to investigate the Wittig reaction mechanism for these substrates were performed. Indeed, we verified the existence of a first high-energy transition state (TS1), leading to planar OPA intermediates (Scheme 6). Significant puckering was observed in TS1 for the (Z)-isomer, reducing unfavourable interactions between the aldehyde and ylide substituents. The latter formation of the alkene undergoes a second transition state (TS2) with the P-C bond's advanced cleavage. Here too, some puckering is observed. Most importantly, this secondary transition state was much lower in energy than TS1, supporting our hypothesis that selectivity is controlled by relative configuration at TS1 and that the reaction is irreversible after this point.

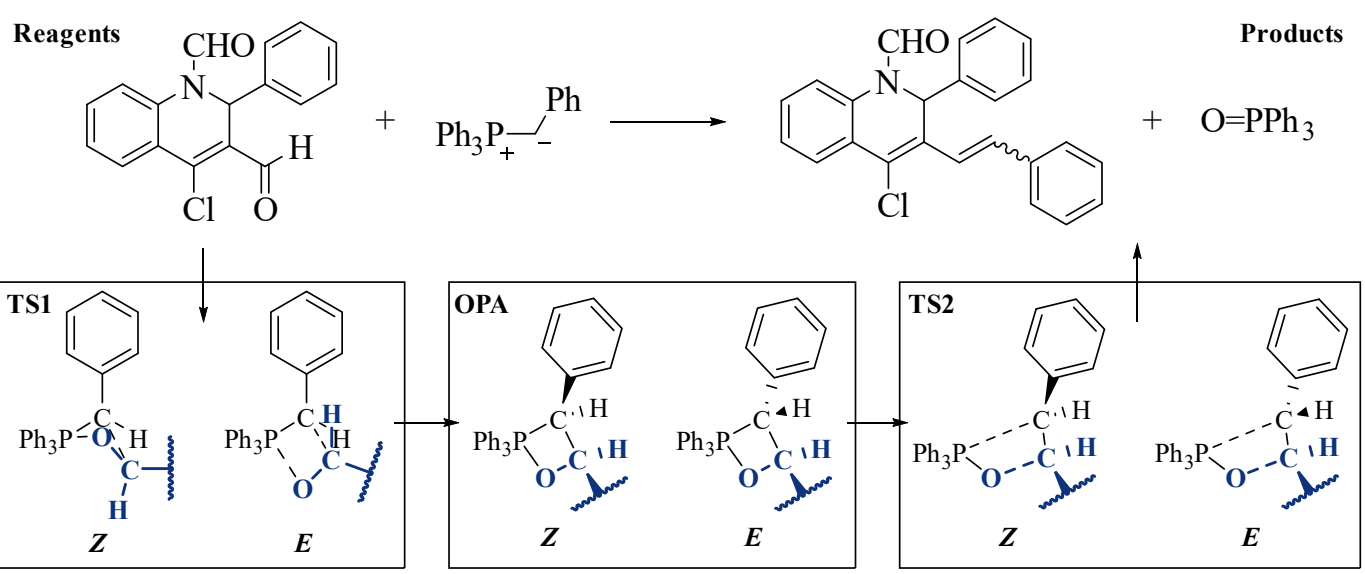

Scheme 6. Preliminary investigation into the mechanism of the Wittig reaction.

Graph 1 shows the calculated potential energy profile for the reaction in study, here using a more precise 6-31G(d) basis set. A similar high-energy transition state preceding phosphoxetane formation was found for both configurations. The transition state TS1, shown in Scheme 6, has a geometry consistent with previous works, with advanced formation of the C-C bond (1.94-2.14 Â) but mainly electrostatic P-O interaction over $3 \hat{A}$. The energy barrier of this rate-determining step of over $10 \mathrm{kcal} / \mathrm{mol}$ is higher than expected. This is most likely due to the complexity of the aldehyde structure, increasing steric constraints in the transition state, and the charge distribution to the conjugated heterocyclic ring.

Comparing both isomers (Figure 1), significantly lower energy was found for the TS leading to the $(E)$-isomer, in clear contradiction with most experimental and computational studies on semi-stabilized ylides but in agreement with our experimental results. This suggests that additional factors may play a role. In both cases, the transition state has an early character $(\mathrm{C}-\mathrm{C}$ bond $\sim 2.1 \hat{\mathrm{A}})$ that reduces steric interactions between substituents.

Furthermore, the non-aromatiC-Nature of the aldehyde substituent allows increased flexibility to reduce any steric constraints. However, the presence of the large aryl substituent on the C-2 sp ${ }^{3}$ carbon limits its ability to assume a puckered state (described as an increase in the PCCO dihedral angle) as it approaches the phosphine phenyl substituents. This is particularly relevant in the (Z)-isomer, where puckering acts as a tool to reduce unfavorable interactions between the aldehyde and both the phosphine and ylide substituents and thus reduce the energy of the transition state. Therefore, the restricted puckering of the (Z)-TS1 may explain its higher energy in our calculations and the preference for the formation of the $E$ quinoline product. 


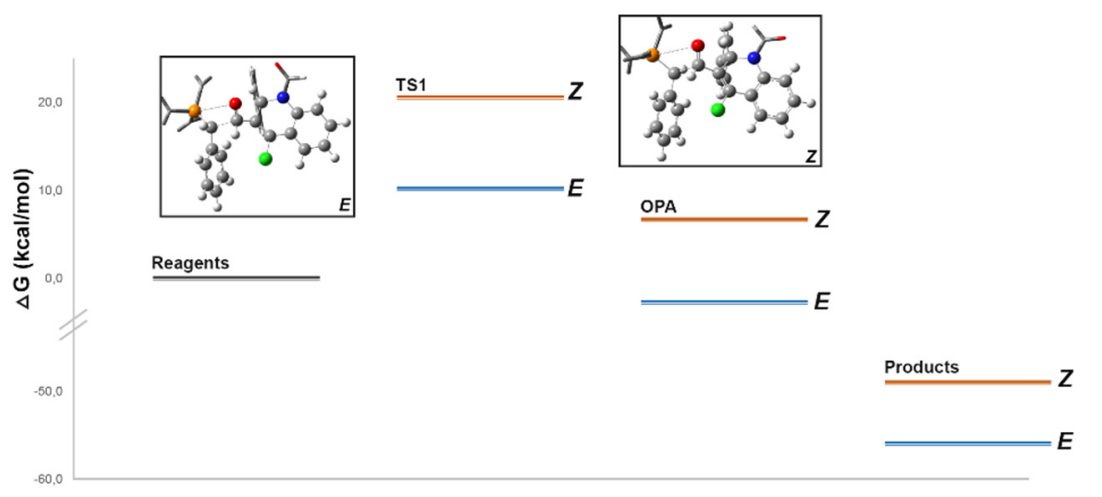

Figure 1. Calculated potential energy surface for the reaction of $2 \mathbf{a}$ with triphenylphosphonium ylide $9 \mathbf{a}$.

\section{Materials and Methods}

\subsection{General Experimental Procedures}

Melting points were determined on a B-545 melting point apparatus (Büchi, Porto, Portugal) and are uncorrected. ${ }^{1} \mathrm{H}$ and ${ }^{13} \mathrm{C}-\mathrm{NMR}$ spectra were recorded on Avance III 300, Avance III HD 500, and Avance III HD $700\left[300.13 \mathrm{MHz}\left({ }^{1} \mathrm{H}\right), 75.47 \mathrm{MHz}\left({ }^{13} \mathrm{C}\right) ; 500.13 \mathrm{MHz}\left({ }^{1} \mathrm{H}\right), 125.76 \mathrm{MHz}\left({ }^{13} \mathrm{C}\right)\right.$; $\left.700.13 \mathrm{MHz}\left({ }^{1} \mathrm{H}\right), 176,05 \mathrm{MHz}\left({ }^{13} \mathrm{C}\right)\right]$ spectrometers (Bruker, Wissembourg, France) with TMS as internal reference and with $\mathrm{CDCl}_{3}$ or $\mathrm{DMSO}_{6}$ as solvent. Chemical shifts $(\delta)$ are reported in parts per million values and coupling constants $(J)$ in Hertz. Unequivocal ${ }^{1} \mathrm{H}$ assignments were made using $2 \mathrm{D}$ NOESY experiments (mixing time of $800 \mathrm{~ms}$ ), while ${ }^{13} \mathrm{C}$ assignments were made based on $2 \mathrm{D}$ gHSQC $\left({ }^{1} \mathrm{H} /{ }^{13} \mathrm{C}\right)$ and gHMBC (delays for one bond and long-range $\mathrm{J}_{\mathrm{C} / \mathrm{H}}$ couplings were optimized for 145 and $7 \mathrm{~Hz}$, respectively) experiments. Positive-ion ESI mass spectra were acquired using a Q-TOF2 instrument (Bruker, Wissembourg, France). Nitrogen was used as nebulizer gas and argon as collision gas. The needle voltage was set at $3000 \mathrm{~V}$, with the ion source at $80^{\circ} \mathrm{C}$ and desolvation temperature at $150^{\circ} \mathrm{C}$. Cone voltage was $35 \mathrm{~V}$. High resolution mass spectra $\left(\mathrm{HRMS}^{\mathrm{ESSI}}{ }^{+}\right)$were measure in a microTOF (focus) mass spectrometer. Ions were generated using an Apollo ll (ESI) source (Bruker, Wissembourg, France). Ionization was achieved by electrospray, using a voltage of $4500 \mathrm{~V}$ applied to the needle and a counter voltage between 100 and $150 \mathrm{~V}$ applied to the capillary. Elemental analyses were obtained with a 932 CHNS analyser (LECO, Madrid, Spain). Preparative thin layer chromatography was carried out with Silica gel $60 \mathrm{GF}_{254}$ (Merck KGaA, Porto, Portugal) and column chromatography using Silica gel 0.060-0.020 mm 60A (Acros Organics, Porto, Portugal). All other chemicals and solvents were obtained from commercial sources and used as received or dried using standard procedures.

\subsection{General Procedure for the Vilsmeyer-Haack Formylation of Flavanones 1a-c and 2-aryl-2,3-} dihydroquinolin-4(1H)-one $\mathbf{1 d - f}$

A mixture of $\mathrm{POCl}_{3}(0.1 \mathrm{~mL}, 1 \mathrm{mmol})$ in dry DMF $(2 \mathrm{~mL})$ was stirred under $0{ }^{\circ} \mathrm{C}$ during $15 \mathrm{~min}$. After that period, a solution of the appropriate 2-aryl-2,3-dihydroquinolin-4(1H)one 1d-f $(0.1 \mathrm{mmol}$ in $\left.10 \mathrm{~mL} \mathrm{CH} \mathrm{Cl}_{2}\right)$ or the appropriate flavanone $1 \mathrm{a}-\mathrm{c}(21 \mathrm{mg}, 0.1 \mathrm{mmol})$ was added and the reaction was stirred at $\sim 60{ }^{\circ} \mathrm{C}$ for $1.5 \mathrm{~h}$ to $2 \mathrm{~h}$ (in the case of 2-aryl-2,3-dihydroquinolin-4(1H)one $1 \mathrm{~d}-\mathbf{f}$ ) or 10 to $12 \mathrm{~h}$ (flavanone $1 \mathbf{a}-\mathbf{c}$ ), depending on the starting material consumption. Then it was poured into a mixture of ice/water $(10 \mathrm{~g} / 15 \mathrm{~mL})$ and the $\mathrm{pH}$ value was adjusted to $5 \mathrm{using}$ a saturated solution of $\mathrm{NaHCO}_{3}$. The solid obtained was filtered, dissolved in dichloromethane $(25 \mathrm{~mL})$ and the organic phase was washed with water $(3 \times 25 \mathrm{~mL})$. The organic phase was dried over anhydrous sodium sulfate and evaporated. The residue was purified by preparative thin layer chromatography using appropriate mixtures of eluent, $\mathrm{CH}_{2} \mathrm{Cl}_{2}$ /hexane.

4-Chloro-2-phenyl-2H-chromene-3-carbaldehyde (2a). Yellow solid, yield (१) 95\%, m. p. 101-103 ${ }^{\circ} \mathrm{C}$. ${ }^{1} \mathrm{H}-\mathrm{NMR}\left(300.13 \mathrm{MHz} \mathrm{CDCl}_{3}\right.$ ): $\delta 6.40$ (s, 1H, H-2); 6.89 (dd, 1H, H-8, J 1.3 and 8.1 Hz); 7.03 (ddd, 1H, H-6, J 1.3, 7.1 and 8.1 Hz); 7.25-7.31 (m, 5H, H-2' $\left.3^{\prime}, 4^{\prime}, 5^{\prime}, 6^{\prime}\right) ; 7.35$ (ddd, 1H, H-7, J 1.3, 7.1 and 8.1 Hz); 
$7.72(\mathrm{dd}, 1 \mathrm{H}, \mathrm{H}-5, J 1.3$ and $8.1 \mathrm{~Hz}) ; 10.30$ (s, $1 \mathrm{H}, 3-\mathrm{CHO}) \mathrm{ppm} .{ }^{13} \mathrm{C}-\mathrm{NMR}\left(75.47 \mathrm{MHz} ; \mathrm{CDCl}_{3}\right): \delta 75.0$ (C-2); 117.4 (C-8); 120.0 (C-4a); 122.0 (C-6); 126.6 (C-5); 126.8 (C-2', ,6'); 128.5 (C-3', 5' $) ; 128.8$ (C-4'); 134.5 (C-7); $138.1\left(\mathrm{C}-1^{\prime}\right), 143.9$ (C-4); 155.1 (C-8a); 188.2 (3-CHO) ppm. ESI ${ }^{+}$-MS m/z (\%): $271.1^{35} \mathrm{Cl}[\mathrm{M}+\mathrm{H}]^{+}$ (36); $273.1{ }^{37} \mathrm{Cl}[\mathrm{M}+\mathrm{H}]^{+}(24) ; 293.1{ }^{35} \mathrm{Cl}[\mathrm{M}+\mathrm{Na}]^{+}(70) ; 295.1{ }^{37} \mathrm{Cl}[\mathrm{M}+\mathrm{Na}]^{+}$(6). EI-HRMS: calcd for $\left(\mathrm{C}_{16} \mathrm{H}_{11} \mathrm{O}_{2}{ }^{35} \mathrm{Cl}\right)$ 270.0448; found 270.0441. Calcd for $\left(\mathrm{C}_{16} \mathrm{H}_{11} \mathrm{O}_{2}{ }^{37} \mathrm{Cl}\right) 272.0418$; found 272.0409.

4-Chloro-2-(4-methoxyphenyl)-2H-chromene-3-carbaldehyde (2b). Yellow solid, $\eta 77 \%$, m. p. 78-80 ${ }^{\circ} \mathrm{C} .{ }^{1} \mathrm{H}-\mathrm{NMR}\left(300.13 \mathrm{MHz}, \mathrm{CDCl}_{3}\right): \delta 3.74\left(\mathrm{~s}, 3 \mathrm{H}, 4^{\prime}-\mathrm{OCH}_{3}\right) ; 6.33(\mathrm{~s}, 1 \mathrm{H}, \mathrm{H}-2) ; 6.79$ (d large, 2H, H-3', $\left.5^{\prime}, J 9.2 \mathrm{~Hz}\right) ; 6,85$ (dd, 1H, H-8, J 1.3 and $8.0 \mathrm{~Hz}$ ); 7.02 (ddd, $1 \mathrm{H}, \mathrm{H}-6, J$ 1.3, 7.1 and $8.0 \mathrm{~Hz}$ ); 7.22 (dlarge, 2H, H-2' ', $6^{\prime}, J 9.2 \mathrm{~Hz}$ ); 7.33 (ddd, 1H, H-7, J 1.3, 7.1 and $8.0 \mathrm{~Hz}$ ); 7.73 (dd, 1H, H-5, J 1.3 and $8.0 \mathrm{~Hz}) ; 10.27$ (s, 1H, 3-CHO) ppm. ${ }^{13} \mathrm{C}-\mathrm{NMR}\left(75.47 \mathrm{MHz} ; \mathrm{CDCl}_{3}\right): \delta 55.2\left(4^{\prime}-\mathrm{OCH}_{3}\right) ; 74.9(\mathrm{C}-2)$; 113.9 (C-3', $\left.5^{\prime}\right) ; 117.5$ (C-8); 120.0 (C-4a); 121.9 (C-6); 126.4 (C-5); 126.9 (C-3); 128.3 (C-2', 6' ); 130.1 (C-1'); 134.4 (C-7); 143.6 (C-4'); 143.9 (C-4); 155.0 (C-8a); 188.1 (3-CHO) ppm. ESI ${ }^{+}-\mathrm{MS}$ m/z (\%): $301.1{ }^{35} \mathrm{Cl}$ [M + $\mathrm{H}]^{+}(5) ; 303.1{ }^{37} \mathrm{Cl}[\mathrm{M}+\mathrm{H}]^{+}(100) ; 323.1{ }^{35} \mathrm{Cl}[\mathrm{M}+\mathrm{Na}]^{+}(40) ; 325.1{ }^{37} \mathrm{Cl}[\mathrm{M}+\mathrm{Na}]^{+}$(2). EI ${ }^{+}-\mathrm{HRMS}$ : calcd for $\left(\mathrm{C}^{17} \mathrm{H}_{13} \mathrm{O}_{3}{ }^{35} \mathrm{Cl}\right) 300.0553$, found 300.0563. Calcd. for $\left(\mathrm{C}_{17} \mathrm{H}_{13} \mathrm{O}_{3}{ }^{37} \mathrm{Cl}\right) 302.0524$, found 302.0527 .

4-Chloro-2-(4-nitrophenyl)-2H-chromene-3-carbaldehyde (2c). Yellow solid, $\eta 66 \%$, m. p. $187-188^{\circ} \mathrm{C}$. ${ }^{1} \mathrm{H}-\mathrm{NMR}\left(300.13 \mathrm{MHz}, \mathrm{CDCl}_{3}\right): \delta 6.47(\mathrm{~s}, 1 \mathrm{H}, \mathrm{H}-2) ; 6.97(\mathrm{dd}, 1 \mathrm{H}, \mathrm{H}-8, J 1.3$ and $8.1 \mathrm{~Hz}) ; 7.08(\mathrm{ddd}, 1 \mathrm{H}$, H-6, J 1.3, 7.1 and 8.1 Hz); 7.42 (ddd, 1H, H-7, J 1.3, 7.1 and 8.1 Hz); 7.47 (dlargo, 2H, H-2', ,' , J 9.0 Hz); 7.74 (dd, 1H, H-5, J 1.3 and $8.1 \mathrm{~Hz}) ; 8.13$ (dlargo, 2H, H-3', 5' , J 9.0 Hz); 10.32 (s, 1H, 3-CHO) ppm. ${ }^{13} \mathrm{C}-\mathrm{NMR}$ (75.47 MHz; $\mathrm{CDCl}_{3}$ ): $\delta 73.8$ (C-2); 117.4 (C-8); 119.0 (C-4a); 122.7 (C-6); 123.8 (C-3', ,5'); 126.0 (C-3); 126.9 (C-5); $127.6\left(\mathrm{C}-2^{\prime}, 6^{\prime}\right), 135.1$ (C-7); 144.5 (C-4); $145.6\left(\mathrm{C}-1^{\prime}\right) ; 147.2\left(\mathrm{C}-4^{\prime}\right) ; 154.6$ (C-8a); 188.1 (3-CHO) ppm. ESI ${ }^{+}-\mathrm{MS}$ $m / z(\%): 316.1{ }^{35} \mathrm{Cl}[\mathrm{M}+\mathrm{H}]^{+}(100) ; 318.1{ }^{37} \mathrm{Cl}[\mathrm{M}+\mathrm{H}]^{+}(40) ; 338.1{ }^{35} \mathrm{Cl}[\mathrm{M}+\mathrm{Na}]^{+}(29) ; 340.1{ }^{37} \mathrm{Cl}[\mathrm{M}+\mathrm{Na}]^{+}$ (3). EI ${ }^{+}$-HRMS: calcd for $\left(\mathrm{C}_{16} \mathrm{H}_{10} \mathrm{NO}_{4}{ }^{35} \mathrm{Cl}\right) 315.0298$, found 315.0304. Calcd for $\left(\mathrm{C}_{16} \mathrm{H}_{10} \mathrm{NO}_{4}{ }^{37} \mathrm{Cl}\right) 317.0269$, found 317.0278.

4-Chloro-2-phenyl-1,2-dihydroquinoline-1,3-dicarbaldehyde (2d). Yellow oil, $\eta 60 \%$. ${ }^{1} \mathrm{H}-\mathrm{NMR}$ (300.13 MHz; $\left.\mathrm{CDCl}_{3}\right): \delta 6.94$ (s, 1H, H-2); 7.09-7.20 (m, 6H, H-2' ,3' , 4' $\left., 5^{\prime}, 6^{\prime}, 8\right) ; 7.36$ (t, 1H, H-6, J 7.6 Hz); 7.49 (t, 1H, H-7, J 7.6 Hz); 8.00 (d, 1H, H-5, J 7.8 Hz); 8.70 (s, 1H, NCHO); 10.32 (s, 1H, 3-CHO) ppm. ${ }^{13} \mathrm{C}-\mathrm{NMR}$ (75.47 MHz; CDCl3): $\delta 50.6$ (C-2); 118.4 (C-8); 126.0 (C-6); 127.1 (C-3', $\left.4^{\prime}\right) ; 127.7$ (C-5); 128.5 (C-4'); 128.7 (C-2', 6'); 131.0 (C-3); 133.2 (C-1'); 133.4 (C-7); 136.8 (C-4a); 137.2 (C-8a); 142.9 (C-4); 160.6 (1-NCHO); $187.8(3-\mathrm{CHO}) \mathrm{ppm}$. MS (ESI $\left.{ }^{+}\right) \mathrm{m} / \mathrm{z}(\%): 320.0{ }^{35} \mathrm{Cl}[\mathrm{M}+\mathrm{Na}]^{+}(100) ; 322.0{ }^{37} \mathrm{Cl}[\mathrm{M}+\mathrm{Na}]^{+}(3)$.

4-Chloro-2-(4-methoxyphenyl)-1,2-dihydroquinoline-1,3-dicarbaldehyde (2e). Yellow oil, $\eta 70 \%$. ${ }^{1} \mathrm{H}-\mathrm{NMR}\left(300.13 \mathrm{MHz} ; \mathrm{CDCl}_{3}\right): \delta 3.71\left(\mathrm{~s}, 3 \mathrm{H}, 4^{\prime}-\mathrm{OCH}_{3}\right) ; 6.72\left(\mathrm{~d}, 2 \mathrm{H}, \mathrm{H}-3^{\prime}, 5^{\prime}, J 8.6 \mathrm{~Hz}\right) ; 6.88(\mathrm{~s}, 1 \mathrm{H}, \mathrm{H}-2)$; $7.12\left(\mathrm{~d}, 2 \mathrm{H}, \mathrm{H}-2^{\prime}, 6^{\prime}, J\right.$ J 8.6 Hz); 7.11-7.15 (m, 1H, H-8); 7.36 (t, 1H, H-6, J 7.7 Hz); 7.48 (t, 1H, H-7, J 7.7 Hz); 8.00 (d, 1H, H-5, J 7.8 Hz); 8.69 (s, 1H, NCHO); 10.30 (s, 1H, CHO) ppm. ${ }^{13} \mathrm{C}-\mathrm{NMR}\left(75.47 \mathrm{MHz} ; \mathrm{CDCl}_{3}\right)$ : $\delta 50.2(\mathrm{C}-2) ; 55.2\left(4^{\prime}-\mathrm{OCH}_{3}\right) ; 114.0\left(\mathrm{C}-3^{\prime}, 5^{\prime}\right) ; 118.4(\mathrm{C}-8) ; 126.0$ (C-6); 127.4 (C-4a); 127.6 (C-5); 128.5 (C-2', 6' $)$; 129.5 (C-1'); 131.7 (C-3); 133.4 (C-7); 136.7 (C-8a); 142.8 (C-4); 159.6 (C-4'); 160.5 (1-NCHO); $187.8(3-\mathrm{CHO}) \mathrm{ppm}$. MS $\left(\mathrm{ESI}^{+}\right) \mathrm{m} / \mathrm{z}(\%): 350.1{ }^{35} \mathrm{Cl}[\mathrm{M}+\mathrm{Na}]^{+}(100) ; 352,1{ }^{37} \mathrm{Cl}[\mathrm{M}+\mathrm{Na}]^{+}(40)$.

4-Chloro-2-(4-nitrophenyl)-1,2-dihydroquinoline-1,3-dicarbaldehyde (2f). Orange solid, $\eta 40 \%$, m.p. $151-153^{\circ} \mathrm{C}^{1}{ }^{1} \mathrm{H}-\mathrm{NMR}\left(300.13 \mathrm{MHz} ; \mathrm{CDCl}_{3}\right): \delta 7.00$ (s, 1H, H-2); 7.19 (d, 1H, H-8, J 7.9 Hz); 7.37 (d, 2H, H-2' , 6' , J $8.8 \mathrm{~Hz}) ; 7.42$ (t, 1H, H-6, J 7.6 Hz); 7.54 (t, 1H, H-7, J 7.6 Hz); 8.03 (d, 1H, H-5, J 7.9 Hz); 8.07 (d, 2H, H-3' , 5' , J $8.8 \mathrm{~Hz}) ; 8.72(\mathrm{~s}, 1 \mathrm{H}, 1-\mathrm{NCHO}) ; 10.33$ (s, 1H, 3-CHO) ppm. ${ }^{13} \mathrm{C}-\mathrm{NMR}(75.47 \mathrm{MHz}$; $\left.\mathrm{CDCl}_{3}\right)$ : $\delta 50.5$ (C-2); 118.4 (C-8); 123.8 (C-4a); 124.0 (C-3', ,5'); 126.5 (C-6); 128.0 (C-5); 128.1 (C-2', 6' $)$; 130.0 (C-3); 134.0 (C-7); 136.3 (C-8a); 144.0 (C-4); 144.1 (C-1'); 147.8 (C-4'); 160.6 (1-NCHO); 187.6 (3-CHO) ppm. MS (ESI $\left.{ }^{+}\right) \mathrm{m} / z(\%): 343.1{ }^{35} \mathrm{Cl}[\mathrm{M}+\mathrm{H}]^{+}(15) ; 345.1{ }^{37} \mathrm{Cl}[\mathrm{M}+\mathrm{H}]^{+}(2) ; 381.3{ }^{35} \mathrm{Cl}[\mathrm{M}+\mathrm{K}]^{+}$ (100); 383,0 ${ }^{37} \mathrm{Cl}[\mathrm{M}+\mathrm{K}]^{+}$(15). EI-HRMS: calcd. for $\left(\mathrm{C}_{17} \mathrm{H}_{11} \mathrm{~N}_{2} \mathrm{O}_{4}{ }^{35} \mathrm{Cl}\right) 342.0407$; found 342.0415. Calcd for $\left(\mathrm{C}_{17} \mathrm{H}_{11} \mathrm{~N}_{2} \mathrm{O}_{4}{ }^{37} \mathrm{Cl}\right) 344.0378$; found 344.0391 .

\subsection{General Procedures for the Synthesis of the Chromene-Indole Hybrids $\mathbf{4 a - c}$}

An excess of indole $3(94 \mathrm{mg}, 0.80 \mathrm{mmol})$ was added to a round bottom flask along with the appropriate 2-aryl-4-chloro-2H-chromene-3-carbaldehyde $2 \mathbf{a}-\mathbf{c}(0.26 \mathrm{mmol})$ and dissolved in DMSO $(2.5 \mathrm{~mL})$. The mixture was stirred and heated to $130^{\circ} \mathrm{C}$ during $24 \mathrm{~h}$. At the end, the reaction 
mixture was poured into a mixture of ice/water $(10 \mathrm{~g} / 20 \mathrm{~mL})$ and liquid-liquid extraction was performed with dichloromethane $(3 \times 30 \mathrm{~mL})$. The organic layer dried over anhydrous sodium sulfate, solvent evaporated and the residue purified by thin layer chromatography using $\mathrm{CH}_{2} \mathrm{Cl}_{2}$ as eluent.

3,3'-[(4-Chloro-2-phenyl-2H-chromen-3-yl)methylene]bis(1H-indole) (4a). Yellow solid, $\eta 79 \%$, m.p. $106-107^{\circ} \mathrm{C} .{ }^{1} \mathrm{H}-\mathrm{NMR}\left(500.13 \mathrm{MHz} ; \mathrm{CDCl}_{3}\right): \delta 5.94$ (s, 1H, H-2" ); 6.25 (s, 1H, 3"-CHCroInd); 6.41 (d, 1H, H-2, J 2.0 Hz); 6.65-6.69 (m, 3H, H-8" , $\left.3^{\prime \prime \prime}, 5^{\prime \prime \prime}\right)$; 6.73-6.80 (m, 3H, H-2 $\left.{ }^{\prime \prime \prime}, 4^{\prime \prime \prime}, 6^{\prime \prime \prime}\right)$; 6.92-7.03 (m, 5H, H-6" , 5, 6, 7, 5'); 7.89 (dt, 1H, H-7" , J 1.6 and 7.8 Hz); $7.17\left(\mathrm{dt}, 1 \mathrm{H}, \mathrm{H}-6^{\prime}, J 1.0\right.$ and $\left.7.9 \mathrm{~Hz}\right)$; 7.25-7.29 (m, 1H, H-4'); 7.37 (d, 1H, H-7' , J 7.9 Hz); 7.50 (d, 1H, H-2' , J 1.5 Hz); 7.53-7.56 (m, 1H, H-4); $7.64\left(\mathrm{dd}, 1 \mathrm{H}, \mathrm{H}-5^{\prime \prime}, J 1.6\right.$ and $\left.7.8 \mathrm{~Hz}\right) ; 10.32(\mathrm{~d}, 1 \mathrm{H}, \mathrm{H}-1) ; 11.10$ (large s, $\left.1 \mathrm{H}, \mathrm{H}-1^{\prime}\right)$ ppm. ${ }^{13} \mathrm{C}-\mathrm{NMR}$ (125.77 MHz; $\mathrm{CDCl}_{3}$ ): $\delta 36.2$ (3"'-CHCroInd); 79.0 (C-2" ); 110.6 (C-7); 111.2 (C-7'); 114.0 (C-3); 115.7 (C-3'); $116.7\left(\mathrm{C}-8^{\prime \prime}\right) ; 119.2$ (2C, C-5, 5'); 119.3 (C-4); 120.6 (C-4'); 121.5 (C-6); 121.8 (C-6" $)$; 122.2 (C-6');

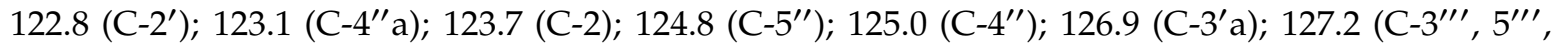

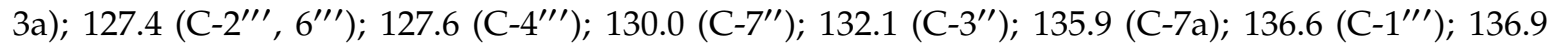
(C-7'a); 152.4 (C-8" a) ppm. ESI ${ }^{+}-\mathrm{MS} m / z$ (\%): $509.2{ }^{35} \mathrm{Cl}\left[\mathrm{M}+\mathrm{Na}^{+}(100) ; 511.2{ }^{37} \mathrm{Cl}[\mathrm{M}+\mathrm{Na}]^{+}(25)\right.$. EI-HRMS: calcd. for $\left(\mathrm{C}_{32} \mathrm{H}_{23} \mathrm{~N}_{2} \mathrm{O}^{35} \mathrm{Cl}\right) 486.1499$; found 486.1490. Calcd for $\left(\mathrm{C}_{32} \mathrm{H}_{23} \mathrm{~N}_{2} \mathrm{O}^{37} \mathrm{Cl}\right) 488.1470$; found 488.1465 .

3,3'-[(4-chloro-2-(4-methoxyphenyl)-2H-chromen-3-yl)methylene]bis( $1 H$-indole) (4b). red solid, $\eta$ 84\%, m.p. $119-121{ }^{\circ} \mathrm{C} .{ }^{1} \mathrm{H}-\mathrm{NMR}\left(500.13 \mathrm{MHz}, \mathrm{DMSO}_{6}\right): \delta 3.50\left(\mathrm{~s}, 3 \mathrm{H}, 4^{\prime \prime \prime}-\mathrm{OCH}_{3}\right) ; 6.01$ (s, $\left.1 \mathrm{H}, \mathrm{H}-2^{\prime \prime}\right)$; 6.15 (s, 1H, 3" -CHCroInd); 6.30 (d, 2H, H-3"' , 5"' , J 8.6 Hz); 6.65 (d, 1H, H-2, J 2.0 Hz); 6.65-6.67 (m, $\left.1 \mathrm{H}, \mathrm{H}-8^{\prime \prime}\right) ; 6.71$ (d, 2H, H-2'"', 6"' , J $\left.8.6 \mathrm{~Hz}\right) ; 6.87$ (t, 1H, H-5', J $\left.7.5 \mathrm{~Hz}\right) ; 6.89-7.01$ (m, 3H, H- 6" , 5, 6); 7.04-7.10 (m, 2H, H-7, 6' $) ; 7.13\left(\mathrm{dt}, 1 \mathrm{H}, \mathrm{H}-7^{\prime \prime}, J 1.0\right.$ and $\left.7.7 \mathrm{~Hz}\right) ; 7.24\left(\mathrm{~d}, 1 \mathrm{H}, \mathrm{H}-4^{\prime}, J\right.$ J $\left.8.0 \mathrm{~Hz}\right) ; 7.35$ (d, 1H, H-2' , J $1.5 \mathrm{~Hz}$ ); 7.41 (d, 1H, H-7' , J $8.1 \mathrm{~Hz}) ; 7.45$ (d, 1H, H-4, J $8.1 \mathrm{~Hz}) ; 7.54$ (dd, 1H, H-5' , J 1.0 and $7.7 \mathrm{~Hz}) ; 10.57$ (d, 1H, H-1, J $2.0 \mathrm{~Hz}) ; 11.06\left(\mathrm{~d}, 1 \mathrm{H}, \mathrm{H}-1^{\prime}, J 1.5 \mathrm{~Hz}\right) \mathrm{ppm} .{ }^{13} \mathrm{C}-\mathrm{NMR}(125.77 \mathrm{MHz}$, DMSO- $\left.d_{6}\right): \delta 35.6$ (3"-CHCroInd); $54.8\left(4^{\prime \prime \prime}-\mathrm{OCH}_{3}\right) ; 77.5$ (C-2" $) ; 111.1$ (C-7); $111.6\left(\mathrm{C}-7^{\prime}\right) ; 112.0$ (C-3)

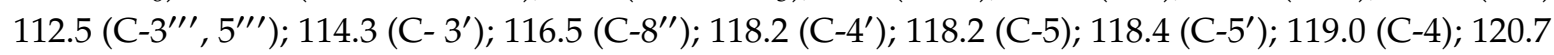

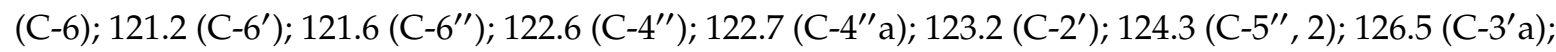

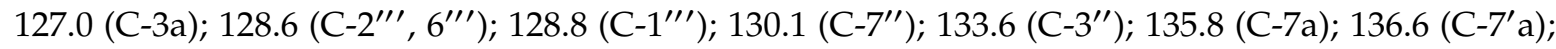
$151.8\left(\mathrm{C}-8^{\prime \prime}\right.$ a); $158.6\left(\mathrm{C}-4^{\prime \prime \prime}\right)$ ppm. ESI ${ }^{+}-\mathrm{MS} \mathrm{m} / z(\%): 539.2{ }^{35} \mathrm{Cl}[\mathrm{M}+\mathrm{Na}]^{+}(29) ; 541.2{ }^{37} \mathrm{Cl}[\mathrm{M}+\mathrm{Na}]^{+}$ (12). EI-HRMS: calcd. for $\left(\mathrm{C}_{33} \mathrm{H}_{25} \mathrm{~N}_{2} \mathrm{O}_{2}{ }^{35} \mathrm{Cl}\right) 516.1605$; found 516.1610. Calcd for $\left(\mathrm{C}_{33} \mathrm{H}_{25} \mathrm{~N}_{2} \mathrm{O}_{2}{ }^{37} \mathrm{Cl}\right)$ 518.1575; found 518.1577.

3,3'-[(4-Chloro-2-(4-nitrophenyl)-2H-chromen-3-yl)methylene]bis( $1 H$-indole) (4c). Yellow solid, $\eta$ $60 \%$, m.p. $179-180{ }^{\circ} \mathrm{C} .{ }^{1} \mathrm{H}-\mathrm{NMR}\left(500.13 \mathrm{MHz}\right.$, DMSO- $\left.d_{6}\right): \delta 6.14$ (s, $\left.1 \mathrm{H}, 3^{\prime \prime}-\mathrm{CHCroInd}\right) ; 6.26(\mathrm{~s}, 1 \mathrm{H}$, H-2" ); 6.69 (dd, 1H, H- 8" , J 0.8 and $8.0 \mathrm{~Hz}) ; 6.75$ (d, 1H, H-2, J 2.1 Hz); 6.82-6.94 (m, 6H, H-2"' , 6" ${ }^{\prime \prime}, 5$, 6, 7, 5' ); 7.03-7.11 (m, 2H, H-6" , 6' $) ; 7.17\left(\mathrm{dt}, 1 \mathrm{H}, \mathrm{H}-7^{\prime \prime}, ~ J 1.6\right.$ and $\left.8.0 \mathrm{~Hz}\right) ; 7.27\left(\mathrm{~d}, 1 \mathrm{H}, \mathrm{H}-4^{\prime}\right.$, , $\left.8.0 \mathrm{~Hz}\right) ; 7.34$ (d, 1H, H-4, J 7.4 Hz); 7.41-7.46 (m, 3H, H-3"' , 5'"' , 7'); 7.56 (d, 1H, H-2' , J 1.6 Hz); 7.62 (dd, 1H, H-5" , $J 1.6$ and $7.8 \mathrm{~Hz}) ; 10.61(\mathrm{~d}, 1 \mathrm{H}, \mathrm{H}-1, J 2.1 \mathrm{~Hz}) ; 11.16\left(\mathrm{~d}, 1 \mathrm{H}, \mathrm{H}-1^{\prime}, J 1.6 \mathrm{~Hz}\right)$ ppm. ${ }^{13} \mathrm{C}-\mathrm{NMR}(125.77 \mathrm{MHz}$, DMSO- $\left.d_{6}\right): \delta 35.7$ (3"-CHCroInd); 77.1 (C-2" $)$; 111.4 (C-7); 112.1 (C-3); 112.1 (C-7'); 113.8 (C-3'); 116.9 $\left(\mathrm{C}-8^{\prime \prime}\right) ; 118.6(\mathrm{C}-4) ; 118.9\left(\mathrm{C}-5,5^{\prime}\right) ; 119.6\left(\mathrm{C}-4^{\prime}\right) ; 121.3$ (C-6); 121.7 (C-6'); $122.2\left(\mathrm{C}-3^{\prime \prime \prime}, 5^{\prime \prime \prime}\right)$; 122.4 (C-6" $)$; 122.6 (C-4" a); 123.3 (C-2'); 123.8 (C-4" ); 125.0 (C-5" ); 125.2 (C-2); 126.9 (C-3'a); 127.1 (C-3a); 128.3

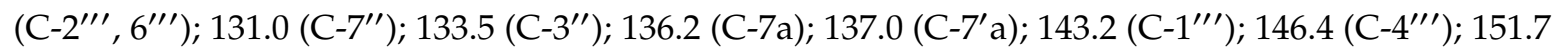
(C-8" a) ppm. ESI ${ }^{+}$-MS m/z (\%): $554.2{ }^{35} \mathrm{Cl}[\mathrm{M}+\mathrm{Na}]^{+}(8) ; 556.2{ }^{37} \mathrm{Cl}[\mathrm{M}+\mathrm{Na}]^{+}$(3). EI-HRMS: calcd. for $\left(\mathrm{C}_{32} \mathrm{H}_{22} \mathrm{~N}_{3} \mathrm{O}_{3}{ }^{35} \mathrm{Cl}\right)$ 531.1350; found 531.1354. Calcd for $\left(\mathrm{C}_{32} \mathrm{H}_{22} \mathrm{~N}_{3} \mathrm{O}_{3}{ }^{37} \mathrm{Cl}\right)$ 533.1320; found 533.1327.

\subsection{General Procedures for the Synthesis of 6-Aryl-6H-chromeno[3,4-c]quinolines 7a-f}

In a round bottom flask with solution of the appropriate 2-aryl-4-chloro-2H-chromene-3carbaldehyde $\mathbf{2 a - c}(0.10 \mathrm{mmol})$ in dichloromethane $(7 \mathrm{~mL})$ was added the correspondent aniline 5 $(0.27 \mathrm{mmol})$. The mixture was stirred at room temperature for 1.5 or $2.5 \mathrm{~h}$ depending on the substituents in the 2-aryl-4-chloro- $2 \mathrm{H}$-chromene-3-carbaldehydes $\mathbf{2 a - c}$. After this period, TFA (2.6 mmol in case of 3,4-dimethoxyanilinine and $26 \mathrm{mmol}$ in case of aniline or 4-nitroaniline) was added to the reaction mixture and left stirring at room temperature for $72 \mathrm{~h}$. Finally, the solvent was evaporated on the rotary 
evaporator and the mixture was purified by preparative chromatography using $\mathrm{CH}_{2} \mathrm{Cl}_{2}$ or a mixture of $\mathrm{CH}_{2} \mathrm{Cl}_{2} /$ hexane (3:1) as eluent.

1-[4-Chloro-2-phenyl-2H-chromen-3-yl)]- $N$-(3,4-dimethoxyphenyl)methanimine (6b). Yellow solid, $\eta 17 \%$, m.p. $79-81^{\circ} \mathrm{C} .{ }^{1} \mathrm{H}-\mathrm{NMR}\left(500.13 \mathrm{MHz}, \mathrm{CDCl}_{3}\right): \delta 3.90$ and $3.91\left(\mathrm{~s}, 3 \mathrm{H}, 3^{\prime \prime}-\right.$ and $\left.4^{\prime \prime}-\mathrm{OCH}_{3}\right) ; 6.79$ (s, 1H, H-2), 6.81 (dd, 1H, H-6" , J 2.3 and $6.0 \mathrm{~Hz}) ; 6.83$ (d, 1H, H- 2" , J $2.3 \mathrm{~Hz}) ; 6.86$ (d, 1H, H-5", J $6.0 \mathrm{~Hz}) ; 6.90(\mathrm{dd}, 1 \mathrm{H}, \mathrm{H}-8, J 1.1$ and $8.1 \mathrm{~Hz}) ; 6.99(\mathrm{dt}, 1 \mathrm{H}, \mathrm{H}-6, J 1.0$ and $7.8 \mathrm{~Hz}) ; 7.41\left(\mathrm{~d}, 2 \mathrm{H}, \mathrm{H}-2^{\prime}, 6^{\prime}\right.$, J $8.8 \mathrm{~Hz}) ; 7.21-7.28\left(\mathrm{~m}, 4 \mathrm{H}, \mathrm{H}-7,3^{\prime}, 4^{\prime}, 5^{\prime}\right) ; 7.63(\mathrm{dd}, 1 \mathrm{H}, \mathrm{H}-5, J 1.5$ and $7.8 \mathrm{~Hz}) ; 8.84(\mathrm{~s}, 1 \mathrm{H}, \mathrm{CHN}) \mathrm{ppm}$. ${ }^{13} \mathrm{C}-\mathrm{NMR}\left(125.77 \mathrm{MHz}, \mathrm{CDCl}_{3}\right)$ : $\delta 56.0$ and 56.1 (3" - and $\left.4^{\prime \prime}-\mathrm{OCH}_{3}\right) ; 76.0(\mathrm{C}-2) ; 105.7\left(\mathrm{C}-6^{\prime \prime}\right) ; 111.2$ (C-5"); 112.9 (C-2" ); 117.2 (C-8); 121.5 (C- 4a); 121.7 (C-6); 125.6 (C-5); 127.3 (C-2', 6'); 127.9 (C-3); 128.3 $\left(\mathrm{C}-3^{\prime}, 4^{\prime}, 5^{\prime}\right) ; 132.2(\mathrm{C}-7) ; 134.8(\mathrm{C}-4) ; 138.8\left(\mathrm{C}-1^{\prime}\right) ; 144.7\left(\mathrm{C}-1^{\prime \prime}\right) ; 148.4\left(\mathrm{C}-4^{\prime \prime}\right) ; 149.3\left(\mathrm{C}-3^{\prime \prime}\right) ; 153.0(\mathrm{CHN})$; 154.2 (C-8a) ppm. ESI+-MS m/z (\%): $406.1{ }^{35} \mathrm{Cl}[\mathrm{M}+\mathrm{H}]^{+}(100) ; 408.1{ }^{37} \mathrm{Cl}[\mathrm{M}+\mathrm{H}]^{+}(52)$.

1-[4-Chloro-2-(4-nitrophenyl)-2H-chromen-3-yl)]- $N$-(3,4-dimethoxyphenyl)methanimine (6f). Yellow solid, $\eta$ 9\%, m.p. $109-112{ }^{\circ} \mathrm{C} .{ }^{1} \mathrm{H}-\mathrm{NMR}\left(300.13 \mathrm{MHz}, \mathrm{CDCl}_{3}\right)$ : $\delta 3.91$ and $3.92\left(\mathrm{~s}, 3 \mathrm{H}, 3^{\prime \prime}-\right.$ and $\left.4^{\prime \prime}-\mathrm{OCH}_{3}\right) ; 6.81\left(\mathrm{t}, 1 \mathrm{H}, \mathrm{H}-2^{\prime \prime}, J 2.0 \mathrm{~Hz}\right) ; 6.84-6.87\left(\mathrm{~m}, 3 \mathrm{H}, \mathrm{H}-2,6^{\prime \prime}, 5^{\prime \prime}\right) ; 6.95(\mathrm{dd}, 1 \mathrm{H}, \mathrm{H}-8, J 1.1$ and $7.9 \mathrm{~Hz}) ; 7.03(\mathrm{dt}, 1 \mathrm{H}, \mathrm{H}-6, J 1.1$ and $7.9 \mathrm{~Hz}) ; 7.30(\mathrm{dt}, 1 \mathrm{H}, \mathrm{H}-7, J 1.6$ and $7.9 \mathrm{~Hz}) ; 7.59\left(\mathrm{~d}, 2 \mathrm{H}, \mathrm{H}-2^{\prime}, 6^{\prime}\right.$, $J 8.8 \mathrm{~Hz}) ; 7.64(\mathrm{dd}, 1 \mathrm{H}, \mathrm{H}-5, J 1.6 \mathrm{and} 7.9 \mathrm{~Hz}) ; 8.11\left(\mathrm{~d}, 1 \mathrm{H}, \mathrm{H}-3^{\prime}, 5^{\prime}, J 8.8 \mathrm{~Hz}\right) ; 8.87$ (s, 1H, CHN) ppm. ${ }^{13} \mathrm{C}-\mathrm{NMR}\left(75.47 \mathrm{MHz}, \mathrm{CDCl}_{3}\right): \delta 56.0$ and $56.1\left(3^{\prime \prime}-\right.$ and $\left.4^{\prime \prime}-\mathrm{OCH}_{3}\right) ; 75.0(\mathrm{C}-2) ; 105.5\left(\mathrm{C}-6^{\prime \prime}\right) ; 111.3\left(\mathrm{C}-5^{\prime \prime}\right)$; 113.2 (C-2' ); 117.1 (C-8); 121.1 (C- 4a); 122.3 (C-6); 123.5 (C-3', 5'); 125.9 (C-5); 127.1 (C-3); 128.1 (C-2', 6' ); 132.7 (C- 7); 135.4 (C-4); 144.1 (C-1" ); $146.1\left(\mathrm{C}-1^{\prime}\right) ; 147.7\left(\mathrm{C}-4^{\prime}\right) ; 148.8\left(\mathrm{C}-3^{\prime \prime}\right) ; 149.5\left(\mathrm{C}-4^{\prime \prime}\right) ; 152.5(\mathrm{CHN}) ;$ 153.7 (C-8a) ppm. ESI ${ }^{+}-\mathrm{MS} \mathrm{m} / z(\%): 451.1^{35} \mathrm{Cl}[\mathrm{M}+\mathrm{H}]^{+}(100) ; 453.1{ }^{37} \mathrm{Cl}[\mathrm{M}+\mathrm{H}]^{+}(30)$.

6-Phenyl-6H-chromeno[3,4-c]quinoline (7a). Yellow oil, $\eta 40 \% .{ }^{1} \mathrm{H}-\mathrm{NMR}\left(500,13 \mathrm{MHz}, \mathrm{CDCl}_{3}\right)$ : $\delta 6.37(\mathrm{~s}, 1 \mathrm{H}, \mathrm{H}-6) ; 7.05$ (dd, 1H, H-4, J 1.3 and $7.9 \mathrm{~Hz}) ; 7.18(\mathrm{dt}, 1 \mathrm{H}, \mathrm{H}-2, J 1.3$ and $7.5 \mathrm{~Hz}) ; 7.39$ (dt, 1H, H-3, J 1.3 and $7.5 \mathrm{~Hz}) ; 7.41-7.50$ (m, 7H, H-7,11, 2' $\left.3^{\prime}, 4^{\prime}, 5^{\prime}, 6^{\prime}\right) ; 7.66$ (d, 1H, H-12, J 8.3 Hz); 7.70 (dt, 1H, H-10, J 1.4 and $7.0 \mathrm{~Hz}) ; 8.15(\mathrm{~d}, 1 \mathrm{H}, \mathrm{H}-9, \mathrm{~J} 8.3 \mathrm{~Hz}) ; 8.50(\mathrm{dd}, 1 \mathrm{H}, \mathrm{H}-1, \mathrm{~J} 1.3$ and $7.9 \mathrm{~Hz}) \mathrm{ppm}$. ${ }^{13} \mathrm{C}-\mathrm{NMR}\left(500,13 \mathrm{MHz}, \mathrm{CDCl}_{3}\right): \delta 80.3$ (C-6); 117.7 (C-4); 122.6 (C-2); 123.2 (C-12c); 125.6 (C-1); 126.3 (C-11); 126.5 (C-6a); 127.5 (C-12a); 127.8 (C-12); 128.2 (C-2', 6'); 128.8 (C-3', 5'); 129.0 (C-4' ); 129.3 (C-9); 129.8 (C-10); 132.0 (C-3); 132.9 (C-7); 138.6 (C-1'); 148.1 (C-8a); 148.9 (C-12b); 156.6 (C-4a) ppm. ESI ${ }^{+}-M S$ $m / z(\%): 310.2[\mathrm{M}+\mathrm{H}]^{+}(42)$.

10,11-Dimethoxy-3-phenyl-3H-chromene[3,4-c]quinoline (7b). Yellow solid, $\eta 63 \%$, m.p. $127-128^{\circ} \mathrm{C}$. ${ }^{1} \mathrm{H}-\mathrm{NMR}\left(500.13 \mathrm{MHz}, \mathrm{CDCl}_{3}\right): \delta 3.95\left(\mathrm{~s}, 3 \mathrm{H}, 11-\mathrm{OCH}_{3}\right) ; 4.07$ (s, 3H, 10-OCH $\left.\mathrm{O}_{3}\right) ; 6.34(\mathrm{~s}, 1 \mathrm{H}, \mathrm{H}-6) ; 6.91$ (s, 1H, H-12); 7.04 (dd, 1H, H-4, J 1.0 and $8.1 \mathrm{~Hz}) ; 7.16(\mathrm{dt}, 1 \mathrm{H}, \mathrm{H}-2, J 1.0$ and $7.6 \mathrm{~Hz}) ; 7.30$ (s, 1H, H-7); 7.35 (ddd, 1H, H-3, J 1.6; 7.6 and $8.1 \mathrm{~Hz}) ; 7.40-7.52\left(\mathrm{~m}, 6 \mathrm{H}, \mathrm{H}-9,2^{\prime}, 3^{\prime}, 4^{\prime}, 5^{\prime}, 6^{\prime}\right) ; 8.45$ (dd, 1H, H-1, J 1.6 and 7.6 Hz) ppm. ${ }^{13} \mathrm{C}-\mathrm{NMR}\left(125.77 \mathrm{MHz}, \mathrm{CDCl}_{3}\right): \delta 56.0\left(11-\mathrm{OCH}_{3}\right) ; 56.2\left(10-\mathrm{OCH}_{3}\right) ; 80.4(\mathrm{C}-6) ; 105.0$ (C-12a); 105.3 (C-12); 107.9 (C-9); 117.6 (C-4); 122.5 (C-2); 123.1 (C-12c); 125.0 (C-1); 126.7 (C-6a); 128.3 (C-2', $\left.6^{\prime}\right) ; 128.8\left(\mathrm{C}-3^{\prime}, 5^{\prime}\right) ; 128.9$ (C-4'); 131.2 (C-3); 131.4 (C-7); 139.0 (C-1'); 145.1 (C-8a); 146.8 (C-12b); 149.7 (C-11); 152.7 (C-10); 156.1 (C-4a) ppm. ESI ${ }^{+}$MS m/z (\%): $370.1[\mathrm{M}+\mathrm{H}]^{+}$(100). EI-HRMS: calcd. for $\left(\mathrm{C}_{24} \mathrm{H}_{19} \mathrm{NO}_{3}\right)$ 369.1365; found 369.1368.

10-Nitro-3-phenyl-3H-chromeno[3,4-c]quinoline (7c). Orange oil, $\eta 25 \% .{ }^{1} \mathrm{H}-\mathrm{NMR}(500,13 \mathrm{MHz}$, $\left.\mathrm{CDCl}_{3}\right): \delta 6.38(\mathrm{~s}, 1 \mathrm{H}, \mathrm{H}-6) ; 7.09$ (d, 1H, H-4, J 8.0 Hz); 7.20-7.26 (m, 1H, H-2); 7.44-7.49 (m, 6H, H-3, $\left.2^{\prime}, 3^{\prime}, 4^{\prime}, 5^{\prime}, 6^{\prime}\right), 7.60(\mathrm{~s}, 1 \mathrm{H}, \mathrm{H}-7) ; 8.26(\mathrm{~d}, 1 \mathrm{H}, \mathrm{H}-9, J 9.1 \mathrm{~Hz}) ; 8.45(\mathrm{dd}, 1 \mathrm{H}, \mathrm{H}-10, J 2.4$ and $9.1 \mathrm{~Hz}) ; 8.55(\mathrm{~d}$, 1H, H-1, J 7.5 Hz); 8.62 (d, 1H, H-12, J $2.4 \mathrm{~Hz})$ ppm. ${ }^{13} \mathrm{C}-\mathrm{NMR}\left(500,13 \mathrm{MHz}, \mathrm{CDCl}_{3}\right): \delta 80.2(\mathrm{C}-6) ; 118.0$ (C-4); 122.2 (C-12a); 122.6 (C-2); 123.1 (C-10); 123.5 (C-6a); 124.6 (C-12); 126.4 (C-1); 128.2 (C-2', 6' ); 128.6 (C-12c); 129.1 (C-3', $\left.5^{\prime}\right) ; 129.4$ (C-4'); 130.8 (C-9); 133.5 (C-3); 134.5 (C-7); 137.8 (C-1'); 145.1 (C-11); 150.2 (C-8a); 152.4 (C-12b); 157.2 (C-4a) ppm. ESI ${ }^{+}$-MS m/z (\%):355.2 [M + H] ${ }^{+}$(100).

3-(4-Methoxyphenyl)-3H-chromeno[3,4-c]quinoline (7d). Yellow oil, $\eta 50 \% .{ }^{1} \mathrm{H}-\mathrm{NMR}(500,13 \mathrm{MHz}$, $\left.\mathrm{CDCl}_{3}\right): \delta 3.84\left(\mathrm{~s}, 3 \mathrm{H}, 4^{\prime}-\mathrm{OCH}_{3}\right) ; 6.32(\mathrm{~s}, 1 \mathrm{H}, \mathrm{H}-6) ; 6.96\left(\mathrm{~d}, 2 \mathrm{H}, \mathrm{H}-3^{\prime}, 5^{\prime}, \mathrm{J} 8.8 \mathrm{~Hz}\right) ; 7.03$ (d, 1H, H-4, J $9.2 \mathrm{~Hz}) ; 7.17$ (t, 1H, H-2, J 7.5 Hz); 7.33-7.50 (m, 5H, H-3, 7, 11, 2' , $\left.6^{\prime}\right) ; 7.65$ (d, 1H, H-12, J 7.8 Hz); 7.69 (t, 1H, H-10, J 7.7 Hz); 8.14 (d, 1H, H-9, J 7.8 Hz); 8.52 (d, 1H, H-1, J 9.2 Hz) ppm. ${ }^{13} \mathrm{C}-\mathrm{NMR}(500,13 \mathrm{MHz}$, $\left.\mathrm{CDCl}_{3}\right): \delta 55.4\left(4^{\prime}-\mathrm{OCH}_{3}\right) ; 80.0(\mathrm{C}-6) ; 114.2\left(\mathrm{C}-3^{\prime}, 5^{\prime}\right) ; 117.7$ (C-4); 122.5 (C-2); 123.5 (C-12c); 125.5 (C-1); 126.2 (C-11); 127.6 (C-12); 127,7 (C-12a); 128.8 (C-12); 129.3 (C-10); 129.7 (C-2' , $\left.6^{\prime}\right) ; 129.7$ (C-1'); 130.8 
(C-1'); 132.0 (C-3); 132.9 (C-7); 148.1 (C-8a); 149.0 (C-12b); 156.5 (C-4a); 160.0 (C-4') ppm. ESI ${ }^{+}$MS m/z (\%): $340.2[\mathrm{M}+\mathrm{H}]^{+}(100)$.

10,11-Dimethoxy-3-(4-methoxyphenyl)-3H-chromene[3,4-c]quinoline (7e). Yellow solid, $\eta 75 \%$, m.p. $107-108{ }^{\circ} \mathrm{C} .{ }^{1} \mathrm{H}-\mathrm{NMR}\left(300.13 \mathrm{MHz}\right.$, acetone- $\left.d_{6}\right): \delta 3.82\left(\mathrm{~s}, 3 \mathrm{H}, 4^{\prime}-\mathrm{OCH}_{3}\right) ; 3.93\left(\mathrm{~s}, 3 \mathrm{H}, 11-\mathrm{OCH}_{3}\right)$; $4.02\left(\mathrm{~s}, 3 \mathrm{H}, 10-\mathrm{OCH}_{3}\right) ; 6.42(\mathrm{~s}, 1 \mathrm{H}, \mathrm{H}-6) ; 6.94-7.02\left(\mathrm{~m}, 3 \mathrm{H}, \mathrm{H}-4,3^{\prime}, 5^{\prime}\right) ; 7.14(\mathrm{dt}, 1 \mathrm{H}, \mathrm{H}-2, \mathrm{~J} 1.1$ and $7.7 \mathrm{~Hz})$; 7.20 (s, 1H, H-12); 7.32-7.43 (m, 3H, H-3,2' , $\left.6^{\prime}\right) ; 7.44$ (s, 1H, H-9); 7.53 (s, 1H, H-7); 8.44 (dd, 1H, H-1, $J 1.6$ and $7.7 \mathrm{~Hz}) \mathrm{ppm} .{ }^{13} \mathrm{C}-\mathrm{NMR}\left(75.47 \mathrm{MHz}\right.$, acetone- $\left.d_{6}\right): \delta 55.5\left(4^{\prime}-\mathrm{OCH}_{3}\right) ; 56.1\left(10-\right.$ and $\left.11-\mathrm{OCH}_{3}\right)$; 80.2 (C-6); 106.4 (C-9); 108.5 (C-12); 114.7 (C-3', 5' ); 118.3 (C-4); 122.8 (C-2); 124.1 (C-12a); 124.5 (C-12c); 125.6 (C-1); 127.3 (C-6a); 130.3 (C- 2',6'); 131.9 (C-3,7); 132.5 (C-1'); 145.9 (C-8a); 146.8 (C-12b); 151.0 (C-11); 154.0 (C-10); 156.9 (C-4a); 160.8 (C-4') ppm. ESI ${ }^{+}-\mathrm{MS} \mathrm{m} / \mathrm{z}$ (\%): 400.1 [M + H] ${ }^{+}$(100). EI-HRMS: calcd. for $\left(\mathrm{C}_{25} \mathrm{H}_{21} \mathrm{NO}_{4}\right)$ 399.1471; found 399.1475.

10,11-Dimethoxy-3-(4-nitrophenyl)-3H-chromene[3,4-c]quinoline (7f). Yellow solid, $\eta 73 \%$, m.p. 149-150 ${ }^{\circ} \mathrm{C} .{ }^{1} \mathrm{H}-\mathrm{NMR}\left(300.13 \mathrm{MHz}, \mathrm{CDCl}_{3}\right): \delta 3.97\left(\mathrm{~s}, 3 \mathrm{H}, 10-\mathrm{OCH}_{3}\right) ; 4.07\left(\mathrm{~s}, 3 \mathrm{H}, 11-\mathrm{OCH}_{3}\right) ; 6.44(\mathrm{~s}, 1 \mathrm{H}$, H-6); 6.93 (s, 1H, H-9); 7.04 (dd, 1H, H-4, J 1.0 and 7.8 Hz); 7.18 (dt, 1H, H-2, J 1.0 and 7.8 Hz); 7.32 (s, 1H, H-7); 7.37 (dt, 1H, H-3, J 1.6 and 7.8 Hz); 7.50 (s, 1H, H-12); 7.65 (d, 2H, H-2', 6', J 8.8 Hz); 8.28 (d, $\left.2 \mathrm{H}, \mathrm{H}-3^{\prime}, 5^{\prime}, J 8.8 \mathrm{~Hz}\right) ; 8.44(\mathrm{dd}, 1 \mathrm{H}, \mathrm{H}-1, J 1.6$ and $7.8 \mathrm{~Hz})$ ppm. ${ }^{13} \mathrm{C}-\mathrm{NMR}\left(75.47 \mathrm{MHz}, \mathrm{CDCl}_{3}\right): \delta 56.1$ (10- $\left.\mathrm{OCH}_{3}\right) ; 56.3\left(11-\mathrm{OCH}_{3}\right) ; 78.9$ (C-6); 105.2 (C-9); 107.9 (C-12); 117.6 (C-4); 123.0 (C-12a); 123.0 (C-2); 123.3 (C-12c); 123.9 (C-3', $\left.5^{\prime}\right) ; 125.0$ (C-6a); 125.1 (C-1); 128.9 (C-2' ,6' $) ; 131.0$ (C-7); 131.6 (C-3); 145.4 (C-8a); 146.1 (C-1'); 146.2 (C-12b); 148.1 (C-4'); 150.0 (C- 10); 153.0 (C-11); 155.3 (C-4a) ppm. ESI ${ }^{+}-\mathrm{MS}$ $m / z(\%): 415.1[\mathrm{M}+\mathrm{H}]^{+}(100)$. EI-HRMS: calcd. for $\left(\mathrm{C}_{24} \mathrm{H}_{18} \mathrm{~N}_{2} \mathrm{O}_{5}\right)$ 414.1216; found 414.1219.

\subsection{General Procedures for the Synthesis of the (Z)-and (E)-2-aryl-4-chloro-3-styryl-2H-chromenes 10a-i/11a-i}

A mixture of appropriate phosphonium halide $(\mathbf{8 a}-\mathbf{c}, 77 \mathrm{mg}, 0.21 \mathrm{mmol})$ in freshly dry THF $(10 \mathrm{~mL})$ and $\mathrm{NaH}(13 \mathrm{mg}, 0.5 \mathrm{mmol})$ was stirred under reflux until formation of the corresponding ylide, then, the 4-chloro-2-phenyl-2H-chromene-3-carbaldehydes $\mathbf{2 a - c}(0.07 \mathrm{mmol})$ was added and the reaction mixture continued in reflux (or room temperature). At the end, the reaction mixture was poured into a mixture of ice $(10 \mathrm{~g})$ and water $(20 \mathrm{~mL})$ and acidified at $\mathrm{pH} \sim 3$ with a solution $\mathrm{HCl}(10 \%)$. Then, extracted with ethyl acetate $(3 \times 20 \mathrm{~mL})$, the organic layer was dried over anhydrous sodium sulphate, the solvent was evaporated to dryness and the solid residue was purified by thin layer chromatography using as eluent a mixtures dichloromethane/hexane.

(Z)-4-Chloro-2-phenyl-3-styryl-2H-chromene (10a). White solid, $\eta 32 \%$, m. p. $112-113{ }^{\circ} \mathrm{C} .{ }^{1} \mathrm{H}-\mathrm{NMR}$ (300.13 MHz; $\left.\mathrm{CDCl}_{3}\right): \delta 5.89$ (s, $\left.1 \mathrm{H}, \mathrm{H}-2\right) ; 6.37$ (d, $\left.1 \mathrm{H}, \mathrm{H}-\alpha, J 12.3 \mathrm{~Hz}\right) ; 6.64(\mathrm{~d}, 1 \mathrm{H}, \mathrm{H}-\beta, J 12.3 \mathrm{~Hz}) ; 6.76(\mathrm{dd}$, $1 \mathrm{H}, \mathrm{H}-8, J 1.3$ and $8.0 \mathrm{~Hz}) ; 6.95(\mathrm{ddd}, 1 \mathrm{H}, \mathrm{H}-6, J 1.3,7.1$ and $8.0 \mathrm{~Hz}) ; 7.14-7.22\left(\mathrm{~m}, 6 \mathrm{H}, \mathrm{H}-2^{\prime}, 3^{\prime}, 4^{\prime}, 5^{\prime}, 6^{\prime}, 7\right)$; 7.31-7.43 (m, 5H, H-2" $\left.,^{\prime \prime}, 4^{\prime \prime}, 5^{\prime \prime}, 6^{\prime \prime}\right) ; 7.55(\mathrm{dd}, 1 \mathrm{H}, \mathrm{H}-5$, J 1.3 and $8.0 \mathrm{~Hz})$ ppm. ${ }^{13} \mathrm{C}-\mathrm{NMR}(75.47 \mathrm{MHz}$; $\mathrm{CDCl}_{3}$ ): $\delta 78.2$ (C-2); 116.4 (C-8); 121.3 (C-4a); 121.4 (C-6); 124.5 (C- $\beta$ ); 124.6 (C-5); 126.8 (C-4); 127.2 $\left(\mathrm{C}-2^{\prime}, 6^{\prime}\right) ; 128.1\left(\mathrm{C}-4^{\prime}\right) ; 128.3\left(\mathrm{C}-3^{\prime}, 5^{\prime}\right) ; 128.4\left(\mathrm{C}-4^{\prime \prime}\right) ; 128.5\left(\mathrm{C}-2^{\prime \prime}, 6^{\prime \prime}\right) ; 128.6\left(\mathrm{C}-3^{\prime \prime}, 5^{\prime \prime}\right) ; 128.7\left(\mathrm{C}-1^{\prime}\right) ; 129.1$

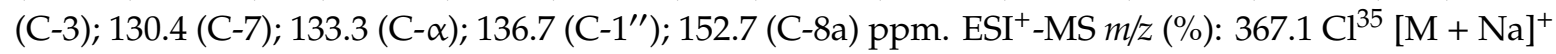
(45), 369.1 $\mathrm{Cl}^{37}[\mathrm{M}+\mathrm{Na}]^{+}$(15). EI-HRMS $\mathrm{m} / \mathrm{z}$ calcd for $\left(\mathrm{C}_{23} \mathrm{H}_{17}{ }^{35} \mathrm{ClO}\right) 344.0968$, found 344.0964. Calcd for $\left(\mathrm{C}_{23} \mathrm{H}_{17}{ }^{37} \mathrm{ClO}\right) 346.0938$, found 346.0933 .

(E)-4-Chloro-2-phenyl-3-styryl-2H-chromene (11a). White solid, $\eta 25 \%$, m. p. $121-123{ }^{\circ} \mathrm{C} .{ }^{1} \mathrm{H}-\mathrm{NMR}$ (300.13 MHz; $\mathrm{CDCl}_{3}$ ): $\delta 6.33$ (s, 1H, H-2); 6.49 (d, $\left.1 \mathrm{H}, \mathrm{H}-\beta, J 16.5 \mathrm{~Hz}\right) ; 6.79$ (dd, $1 \mathrm{H}, \mathrm{H}-8, J 1.3$ and $8.0 \mathrm{~Hz}$ ); 6.94 (ddd, 1H, H-6, J 1.3, 7.1 and 8.0 Hz); 7.13 (ddd, 1H, H-7, J 1.3, 7.1 and 8.0 Hz); 7.25-7.34 (m, 6H, $\left.\mathrm{H}-2^{\prime \prime}, 3^{\prime \prime}, 4^{\prime \prime}, 5^{\prime \prime}, 6^{\prime \prime}, 4^{\prime}\right) ; 7.39-7.45\left(\mathrm{~m}, 4 \mathrm{H}, \mathrm{H}-2^{\prime}, 3^{\prime}, 5^{\prime}, 6^{\prime}\right) ; 7.56(\mathrm{~d}, 1 \mathrm{H}, \mathrm{H}-\alpha, J 16.5 \mathrm{~Hz}) ; 7.57$ (dd, 1H, H-5, J 1.3 and $8.0 \mathrm{~Hz})$ ppm. ${ }^{13} \mathrm{C}-\mathrm{NMR}\left(75.47 \mathrm{MHz} ; \mathrm{CDCl}_{3}\right): \delta 77.4$ (C-2); 116.7 (C-8); 121.7 (C-6); 122.2 (C-4a); $123.2(\mathrm{C}-\alpha) ; 125.0(\mathrm{C}-5) ; 126.8\left(\mathrm{C}-2^{\prime \prime}, 6^{\prime \prime}\right) ; 127.2(\mathrm{C}-4) ; 127.7\left(\mathrm{C}-2^{\prime}, 6^{\prime}\right) ; 128.3\left(\mathrm{C}-4^{\prime}\right) ; 128.5\left(\mathrm{C}-3^{\prime \prime}, 5^{\prime \prime}\right) ; 128.7$ $\left(\mathrm{C}-3^{\prime}, 5^{\prime}\right) ; 130.4$ (C-7); $131.8(\mathrm{C}-\beta) ; 136.7$ (C-3); 137.7 (C-1'); 152.2 (C-8a) ppm.

(Z)-4-Chloro-3-(4-ethoxyvinyl)-2-phenyl-2H-chromene (10b). White solid, $\eta 45 \%$, m. p. $100-102{ }^{\circ} \mathrm{C}$. ${ }^{1} \mathrm{H}-\mathrm{NMR}\left(300.13 \mathrm{MHz} ; \mathrm{CDCl}_{3}\right): \delta 1.42\left(\mathrm{t}, 3 \mathrm{H}, 4^{\prime \prime}-\mathrm{OCH}_{2} \mathrm{CH}_{3}, J 7.0 \mathrm{~Hz}\right) ; 4.05\left(\mathrm{qd}, 2 \mathrm{H}, 4^{\prime}-\mathrm{OCH}_{2} \mathrm{CH}_{3}\right.$, J 1.7 and $7.0 \mathrm{~Hz}) ; 5.95$ (s, 1H, H-2); 6.24 (d, $1 \mathrm{H}, \mathrm{H}-\alpha, J 12.2 \mathrm{~Hz}) ; 6.58(\mathrm{~d}, 1 \mathrm{H}, \mathrm{H}-\beta, J 12.2 \mathrm{~Hz}) ; 6.77(\mathrm{dd}, 1 \mathrm{H}$, 
H-8, J 1.1 and $7.8 \mathrm{~Hz}) ; 6.87$ (d, 2H, H-3" ,5" , J 9.0 Hz); 6.95 (dt, 1H, H-6, J 1.1 and 7.6 Hz); 7.15 (dt, 1H, H-7, J 1.1 and 7.6 Hz); 7.19-7.23 (m, 5H, H-2', $\left.3^{\prime}, 4^{\prime}, 5^{\prime}, 6^{\prime}\right) ; 7.37$ (d, 2H, H-2" , 6" , J 9.0 Hz); 7.54 (dd, 1H, $\mathrm{H}-5$, J 1.1 and $7.8 \mathrm{~Hz}) \mathrm{ppm} .{ }^{13} \mathrm{C}-\mathrm{NMR}\left(75.47 \mathrm{MHz} ; \mathrm{CDCl}_{3}\right): \delta 14.8\left(4^{\prime \prime}-\mathrm{OCH}_{2} \mathrm{CH}_{3}\right) ; 63.4\left(4^{\prime \prime}-\mathrm{OCH}_{2} \mathrm{CH}_{3}\right)$; 78.2 (C-2); 114.4 (C-3", $\left.5^{\prime \prime}\right) ; 116.3$ (C-8); 121.4 (C-6); 121.5 (C-4a); 122.7 (C- $\left.\alpha\right) ; 124.5$ (C-5); 126.3 (C-4); $127.2\left(\mathrm{C}-2^{\prime}, 6^{\prime}\right) ; 128.2\left(\mathrm{C}-3^{\prime}, 5^{\prime}\right) ; 128.4\left(\mathrm{C}-4^{\prime}\right) ; 129.1\left(\mathrm{C}-1^{\prime}\right) ; 129.5(\mathrm{C}-3) ; 130.1\left(\mathrm{C}-2^{\prime \prime}, 6^{\prime \prime}\right) ; 130.2(\mathrm{C}-7) ; 132.9$ (C- $\beta) ; 138.9\left(\mathrm{C}-1^{\prime \prime}\right) ; 152.6$ (C-8a); $158.8\left(\mathrm{C}-4^{\prime \prime}\right)$ ppm. ESI $^{+}-\mathrm{MS} \mathrm{m} / \mathrm{z}(\%): 411.1 \mathrm{Cl}^{35}\left[\mathrm{M}+\mathrm{Na}^{+}(100)\right.$; $413.1 \mathrm{Cl}^{37}[\mathrm{M}+\mathrm{Na}]^{+}$(3). EI-HRMS $m / z$ calcd for $\left(\mathrm{C}_{25} \mathrm{H}_{21}{ }^{35} \mathrm{ClO}_{2}\right) 388.1230$, found 388.1226. Calcd for $\left(\mathrm{C}_{25} \mathrm{H}_{21}{ }^{37} \mathrm{ClO}_{2}\right)$ 390.1201, found 390.1216.

(E)-4-Chloro-3-(4-ethoxyvinyl)-2-phenyl-2H-chromene (11b). Yellow solid, $\eta 25 \%$, m. p. $166-167^{\circ} \mathrm{C}$. ${ }^{1} \mathrm{H}-\mathrm{NMR}\left(300.13 \mathrm{MHz} ; \mathrm{CDCl}_{3}\right): \delta 1.41\left(\mathrm{t}, 3 \mathrm{H}, 4^{\prime \prime}-\mathrm{OCH}_{2} \mathrm{CH}_{3}, J 7.0 \mathrm{~Hz}\right) ; 4.02\left(\mathrm{q}, 2 \mathrm{H}, 4^{\prime}-\mathrm{OCH}_{2} \mathrm{CH}_{3}, J 7.0 \mathrm{~Hz}\right)$; 6.31 (s, 1H, H-2); 6.44 (d, 1H, H- $\beta, J 16.5$ Hz); 6.78 (d largo, 1H, H-8, J 8.0 Hz); 6.85 (d, 2H, H-3" , , $5^{\prime \prime}$, J $9.1 \mathrm{~Hz}) ; 6.94(\mathrm{dt}, 1 \mathrm{H}, \mathrm{H}-6, J 1.4$ and $7.7 \mathrm{~Hz}) ; 7.11(\mathrm{dt}, 1 \mathrm{H}, \mathrm{H}-7, J 1.4$ and $7.7 \mathrm{~Hz}) ; 7.26-7.29(\mathrm{~m}, 3 \mathrm{H}$,

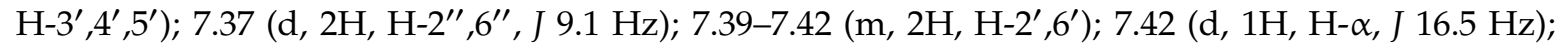
$7.55(\mathrm{dd}, 1 \mathrm{H}, \mathrm{H}-5, J 1.4$ and $8.0 \mathrm{~Hz}) \mathrm{ppm} .{ }^{13} \mathrm{C}-\mathrm{NMR}\left(75.47 \mathrm{MHz} ; \mathrm{CDCl}_{3}\right): \delta 14.8\left(4^{\prime \prime}-\mathrm{OCH}_{2} \mathrm{CH}_{3}\right) ; 63.5$ $\left(4^{\prime \prime}-\mathrm{OCH}_{2} \mathrm{CH}_{3}\right) ; 77.1$ (C-2); $114.7\left(\mathrm{C}-3^{\prime \prime}, 5^{\prime \prime}\right) ; 116.6$ (C-8); 121.0 (C- $\left.\alpha\right) ; 121.6$ (C-6); 122.4 (C-4a); 124.9 (C-5); 125.8 (C-4); $127.7\left(\mathrm{C}-3^{\prime}, 5^{\prime}\right) ; 127.8$ (C-3); $128.1\left(\mathrm{C}-2^{\prime \prime}, 6^{\prime \prime}\right) ; 128.4\left(\mathrm{C}-1^{\prime \prime}\right) ; 128.5\left(\mathrm{C}-2^{\prime}, 6^{\prime}\right) ; 128.6\left(\mathrm{C}-4^{\prime}\right) ; 129.4$ (C-1'); 130.0 (C-7); 131.5 (C- $\beta) ; 137.8$ (C-3); 152.1 (C-8a); 159.3 (C-4") ppm.

(Z)-4-Chloro-2-phenyl-3-(4-nitrovinyl)-2H-chromene (10c). Yellow solid, $\eta 41 \%$, m. p. $159-161{ }^{\circ} \mathrm{C}$. ${ }^{1} \mathrm{H}-\mathrm{NMR}\left(300.13 \mathrm{MHz} ; \mathrm{CDCl}_{3}\right): \delta 5.78(\mathrm{~s}, 1 \mathrm{H}, \mathrm{H}-2) ; 6.53(\mathrm{~d}, 1 \mathrm{H}, \mathrm{H}-\alpha, J 12.2 \mathrm{~Hz}) ; 6.66(\mathrm{~d}, 1 \mathrm{H}, \mathrm{H}-\beta$, $J 12.2 \mathrm{~Hz}) ; 6.79(\mathrm{dd}, 1 \mathrm{H}, \mathrm{H}-8, J 1.2$ and $7.9 \mathrm{~Hz}) ; 6.97(\mathrm{dt}, 1 \mathrm{H}, \mathrm{H}-6, J 1.2$ and $7.6 \mathrm{~Hz}) ; 7.16-7.25(\mathrm{~m}, 6 \mathrm{H}$, $\left.\mathrm{H}-7,2^{\prime}, 3^{\prime}, 4^{\prime}, 5^{\prime}, 6^{\prime}\right) ; 7.53$ (d, 2H, H-2' , 6" ${ }^{\prime \prime}$, J $\left.8.8 \mathrm{~Hz}\right) ; 7.55$ (d, 1H, H-5, J $\left.7.9 \mathrm{~Hz}\right) ; 8.17$ (d, 2H, H-3" , 5" , J $8.8 \mathrm{~Hz}$ ) ppm. ${ }^{13} \mathrm{C}-\mathrm{NMR}\left(75.47 \mathrm{MHz} ; \mathrm{CDCl}_{3}\right): \delta 78.5$ (C-2); 116.5 (C-8); 121.7 (C-6); 121.9 (C-4a); $123.8\left(\mathrm{C}-3^{\prime \prime}, 5^{\prime \prime}\right) ; 125.5$ (C-5); $127.2\left(\mathrm{C}-2^{\prime}, 6^{\prime}\right) ; 127.6$ (C-4); 128.3 (C- $\left.\beta\right) ; 128.5\left(\mathrm{C}-3^{\prime}, 5^{\prime}\right) ; 128.7\left(\mathrm{C}-4^{\prime}\right) ; 129.0$ $\left(\mathrm{C}-1^{\prime}\right) ; 129.2\left(\mathrm{C}-2^{\prime \prime}, 6^{\prime \prime}\right) ; 130.9$ (C- $\left.\alpha\right) ; 131.0$ (C-7); 138.2 (C-3); 143.3 (C-1" ); 147.0 (C-4" ); 152.4 (C-8a) ppm. $\mathrm{ESI}^{+}-\mathrm{MS} m / z(\%): 413.3 \mathrm{Cl}^{35}\left[\mathrm{M}+\mathrm{Na}^{+}(100), 415.3 \mathrm{Cl}^{37}[\mathrm{M}+\mathrm{Na}]^{+}(30)\right.$. Anal. Calcd for $\mathrm{C}_{23} \mathrm{H}_{16} \mathrm{ClNO}_{3}$ : C, $70,86 \% ; \mathrm{H}, 4,14 \% ; \mathrm{N}, 3,59 \%$. Found: C, $71,19 \% ; \mathrm{H}, 4,16 \% ; \mathrm{N}, 3,53 \%$.

(E)-4-Chloro-2-phenyl-3-(4-nitrovinyl)-2H-chromene (11c). Yellow solid, $\eta 33 \%$, m. p. $194-196{ }^{\circ} \mathrm{C}$. ${ }^{1} \mathrm{H}-\mathrm{NMR}\left(300.13 \mathrm{MHz} ; \mathrm{CDCl}_{3}\right): \delta 6.32(\mathrm{~s}, 1 \mathrm{H}, \mathrm{H}-2) ; 6.50(\mathrm{~d}, 1 \mathrm{H}, \mathrm{H}-\beta, J 16.5 \mathrm{~Hz}) ; 6.80(\mathrm{dd}, 1 \mathrm{H}, \mathrm{H}-8$, $J 1.3$ and $8.0 \mathrm{~Hz}) ; 6.97(\mathrm{ddd}, 1 \mathrm{H}, \mathrm{H}-6, J 1.3,7.2$ and $8.0 \mathrm{~Hz}$ ); 7.18 (ddd, $1 \mathrm{H}, \mathrm{H}-7, J$ J 1.3, 7.2 and $8.0 \mathrm{~Hz}$ );

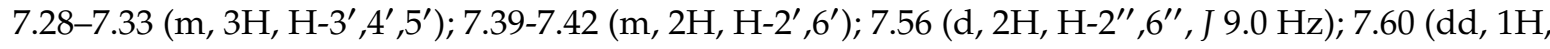
$\mathrm{H}-5, J 1.3$ and $8.0 \mathrm{~Hz}$ ); 7.71 (d, $1 \mathrm{H}, \mathrm{H}-\alpha, J 16.5 \mathrm{~Hz}) ; 8.18$ (d, 2H, H-3" , $5^{\prime \prime}$, , $\left.9.0 \mathrm{~Hz}\right)$ ppm. ${ }^{13} \mathrm{C}-\mathrm{NMR}$ (75.47 MHz; $\mathrm{CDCl}_{3}$ ): $\delta 77.2$ (C-2); 116.9 (C-8); 121.7 (C-4a); 121.9 (C-6); 124.1 (C-3", ,5"); 125.5 (C-5); $126.4(\mathrm{C}-4) ; 127.2\left(\mathrm{C}-2^{\prime \prime}, 6^{\prime \prime}\right) ; 127.3(\mathrm{C}-\alpha) ; 127.6\left(\mathrm{C}-2^{\prime}, 6^{\prime}\right) ; 128.7\left(\mathrm{C}-3^{\prime}, 5^{\prime}\right) ; 128.8\left(\mathrm{C}-4^{\prime}\right) ; 129.0(\mathrm{C}-\beta) ; 129.2$ (C-1'); 131.0 (C-7); 137.2 (C-3); 143.1 (C-1"); 146.8 (C-4"); 152.5 (C-8a) ppm.

(Z)-4-Chloro-2-(4-methoxyphenyl)-3-vinyl-2H-chromene (10d). Yellow solid, $\eta 50 \%$, m. p. $78-80^{\circ} \mathrm{C}$. ${ }^{1} \mathrm{H}-\mathrm{NMR}\left(300.13 \mathrm{MHz} ; \mathrm{CDCl}_{3}\right): \delta 3.73\left(\mathrm{~s}, 3 \mathrm{H}, 4{ }^{\prime}-\mathrm{OCH}_{3}\right) ; 5.83(\mathrm{~s}, 1 \mathrm{H}, \mathrm{H}-2) ; 6.35$ (d, 1H, H- $\left.\alpha, J 12.2 \mathrm{~Hz}\right)$; $6.63(\mathrm{~d}, 1 \mathrm{H}, \mathrm{H}-\beta, J 12.2 \mathrm{~Hz}) ; 6.72$ (dlargo, $1 \mathrm{H}, \mathrm{H}-8, J 8.0 \mathrm{~Hz}) ; 6.73$ (d, 2H, H-3', 5' , J 8.9 Hz); 6.95 (dt, 1H, H-6, J 1.2 and 7.6 Hz); 7.06 (d, 2H, H-2', 6' , J 8.9 Hz); 7.11 (dt, 1H, H-7, J 1.2 and 7.6 Hz); 7.29-7.42 (m, $\left.5 \mathrm{H}, \mathrm{H}-2^{\prime \prime}, 3^{\prime \prime}, 4^{\prime \prime}, 5^{\prime \prime}, 6^{\prime \prime}\right) ; 7.55$ (dd, $1 \mathrm{H}, \mathrm{H}-5, \mathrm{~J} 1.2$ and $\left.8.0 \mathrm{~Hz}\right)$ ppm. ${ }^{13} \mathrm{C}-\mathrm{NMR}\left(75.47 \mathrm{MHz} ; \mathrm{CDCl}_{3}\right): \delta 55.1$

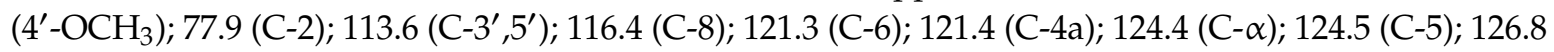
(C-4); 128.1 (C-4" ); $128.5\left(\mathrm{C}-3^{\prime \prime}, 5^{\prime \prime}\right) ; 128.6\left(\mathrm{C}-2^{\prime \prime}, 6^{\prime \prime}\right) ; 128.7$ (C-2',6'); 129.3 (C-3); 130.3 (C-7); 130.8 (C-1'); 133.3 (C- $\beta) ; 136.7\left(\mathrm{C}-1^{\prime \prime}\right) ; 152.5$ (C-8a); $159.7\left(\mathrm{C}-4^{\prime}\right)$ ppm. ESI ${ }^{+}-\mathrm{MS} \mathrm{m} / z(\%): 413.1 \mathrm{Cl}^{35}[\mathrm{M}+\mathrm{K}]^{+}(10)$. EI-HRMS $m / z$ calcd for $\left(\mathrm{C}_{24} \mathrm{H}_{19}{ }^{35} \mathrm{ClO}_{2}\right) 374.1074$; found 374.1082. Calcd for $\left(\mathrm{C}_{24} \mathrm{H}_{19}{ }^{37} \mathrm{ClO}_{2}\right) 376.1044$; found 376.1050 .

(E)-4-Chloro-2-(4-methoxyphenyl)-3-vinyl-2H-chromene (11d). White solid, $\eta 28 \%$, m. p. $187-189^{\circ} \mathrm{C}$. ${ }^{1} \mathrm{H}-\mathrm{NMR}\left(300.13 \mathrm{MHz} ; \mathrm{CDCl}_{3}\right.$ ): $\delta 3.73$ (s, 3H, 4'-OCH ( $_{3}$; 6.28 (s, 1H, H-2); 6.47 (d, $\left.1 \mathrm{H}, \mathrm{H}-\beta, J 16.5 \mathrm{~Hz}\right)$; $6.77(\mathrm{dd}, 1 \mathrm{H}, \mathrm{H}-8, J 1.4$ and $8.3 \mathrm{~Hz}) ; 6.80\left(\mathrm{~d}, 2 \mathrm{H}, \mathrm{H}-3^{\prime}, 5^{\prime}\right.$, , J $\left.8.7 \mathrm{~Hz}\right) ; 6.95(\mathrm{dt}, 1 \mathrm{H}, \mathrm{H}-6, J 1.4$ and $7.6 \mathrm{~Hz})$;

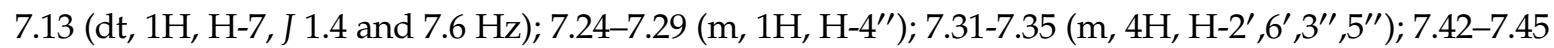
$\left(\mathrm{m}, 2 \mathrm{H}, \mathrm{H}-2^{\prime \prime}, 6^{\prime \prime}\right) ; 7.44\left(\mathrm{~d}, 2 \mathrm{H}, \mathrm{H}-2^{\prime}, 6^{\prime}, J 8.7 \mathrm{~Hz}\right) ; 7.53(\mathrm{~d}, 1 \mathrm{H}, \mathrm{H}-\alpha, J 16.5 \mathrm{~Hz}) ; 7.58$ (dd, 1H, H-5, J 1.4 and $8.3 \mathrm{~Hz}$ ) ppm. ${ }^{13} \mathrm{C}-\mathrm{NMR}\left(75.47 \mathrm{MHz} ; \mathrm{CDCl}_{3}\right): \delta 55.1\left(4^{\prime}-\mathrm{OCH}_{3}\right) ; 76.9$ (C-2); $113.9\left(\mathrm{C}-3^{\prime}, 5^{\prime}\right) ; 116.7$ (C-8); 
121.6 (C-6); 122.2 (C-4a); 123.0 (C- $\alpha) ; 125.0$ (C-5); 126.8 (C-2" , $\left.6^{\prime \prime}\right) ; 127.4$ (C-4); 128.3 (C-4"); 128.5 (C-3); 128.7

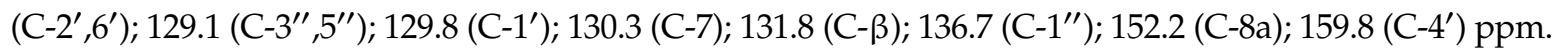

(Z)-4-Chloro-3-(4-ethoxyvinyl)-2-(4-methoxyphenyl)-2H-chromene (10e). Yellow oil, $\eta 30 \%$. ${ }^{1} \mathrm{H}-\mathrm{NMR}\left(300.13 \mathrm{MHz} ; \mathrm{CDCl}_{3}\right): \delta 1.43\left(\mathrm{t}, 3 \mathrm{H}, 4^{\prime \prime}-\mathrm{OCH}_{2} \mathrm{CH}_{3}, J 7.0 \mathrm{~Hz}\right) ; 3.73\left(\mathrm{~s}, 1 \mathrm{H}, 44^{\prime}-\mathrm{OCH}_{3}\right) ; 4.05(\mathrm{q}$, $\left.2 \mathrm{H}, 4^{\prime \prime}-\mathrm{OCH}_{2} \mathrm{CH}_{3}, J 7.0 \mathrm{~Hz}\right) ; 5.89(\mathrm{~s}, 1 \mathrm{H}, \mathrm{H}-2) ; 6.22(\mathrm{~d}, 1 \mathrm{H}, \mathrm{H}-\alpha, J 12.1 \mathrm{~Hz}) ; 6.56(\mathrm{~d}, 1 \mathrm{H}, \mathrm{H}-\beta, J 12.1 \mathrm{~Hz})$; $6.72-6.75\left(\mathrm{~m}, 3 \mathrm{H}, \mathrm{H}-8,3^{\prime}, 5^{\prime}\right) ; 6.87\left(\mathrm{~d}, 2 \mathrm{H}, \mathrm{H}-3^{\prime \prime}, 5^{\prime \prime}\right.$, J $\left.8.9 \mathrm{~Hz}\right) ; 6.95(\mathrm{dt}, 1 \mathrm{H}, \mathrm{H}-6, J 1.4$ and $7.7 \mathrm{~Hz}) ; 7.09(\mathrm{~d}$, 2H, H-2' ,6' , J 8.9 Hz); 7.14 (dt, 1H, H-7, J 1.4 and $7.7 \mathrm{~Hz}) ; 7.36\left(\mathrm{~d}, 2 \mathrm{H}, \mathrm{H}-2^{\prime \prime}, 6^{\prime \prime}\right.$, J $\left.8.9 \mathrm{~Hz}\right) ; 7.55$ (dd,1H, $\mathrm{H}-5, J 1.4$ and $8.0 \mathrm{~Hz})$ ppm. ${ }^{13} \mathrm{C}-\mathrm{NMR}\left(75.47 \mathrm{MHz} ; \mathrm{CDCl}_{3}\right): \delta 14.8\left(4^{\prime \prime}-\mathrm{OCH}_{2} \mathrm{CH}_{3}\right) ; 55.1\left(4^{\prime}-\mathrm{OCH}_{3}\right)$; $63.4\left(4^{\prime \prime}-\mathrm{OCH}_{2} \mathrm{CH}_{3}\right) ; 77.9$ (C-2); $113.6\left(\mathrm{C}-3^{\prime}, 5^{\prime}\right) ; 114.6$ (C-3" , 5" $)$; 116.4 (C-8); 121.3 (C-6); 121.4 (C-4a);

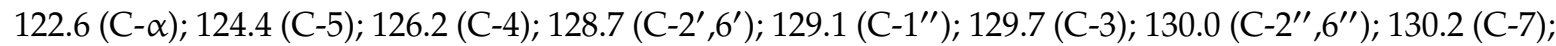
130.9 (C-1'); 132.9 (C- $\beta$ ); 152.5 (C-8a); 158.8 (C-4" ); 159.6 (C-4') ppm. ESI ${ }^{+}-\mathrm{MS} \mathrm{m} / z$ (\%): $441.2 \mathrm{Cl}^{35}$ $[\mathrm{M}+\mathrm{Na}]^{+}(10) ; 443.2 \mathrm{Cl}^{37}[\mathrm{M}+\mathrm{Na}]^{+}(10)$. Anal. Calcd for $\mathrm{C}_{26} \mathrm{H}_{23} \mathrm{ClO}_{3}: \mathrm{C}, 74.55 \% ; \mathrm{H}, 5.53 \%$. Found $\mathrm{C}$, $74.73 \% ; \mathrm{H}, 5.55 \%$.

(E)-4-Chloro-3-(4-ethoxyvinyl)-2-(4-methoxyphenyl)-2H-chromene (11e). Yellow solid, $\eta 20 \%$, m. p. $140-142{ }^{\circ} \mathrm{C} .{ }^{1} \mathrm{H}-\mathrm{NMR}\left(300.13 \mathrm{MHz} ; \mathrm{CDCl}_{3}\right): \delta 1.41\left(\mathrm{t}, 3 \mathrm{H}, 4^{\prime \prime}-\mathrm{OCH}_{2} \mathrm{CH}_{3}, J 7.0 \mathrm{~Hz}\right) ; 3.73(\mathrm{~s}, 1 \mathrm{H}$, $\left.4^{\prime}-\mathrm{OCH}_{3}\right) ; 4.03\left(\mathrm{q}, 2 \mathrm{H}, 4^{\prime \prime}-\mathrm{OCH}_{2} \mathrm{CH}_{3}, J 7.0 \mathrm{~Hz}\right) ; 6.26(\mathrm{~s}, 1 \mathrm{H}, \mathrm{H}-2) ; 6.42(\mathrm{~d}, 1 \mathrm{H}, \mathrm{H}-\beta, J 16.5 \mathrm{~Hz}) ; 6.76$ (dd, $1 \mathrm{H}, \mathrm{H}-8, J 1.3$ and $8.0 \mathrm{~Hz}) ; 6.79$ (d, 2H, H-3' $\left.5^{\prime}, J 8.8 \mathrm{~Hz}\right) ; 6.84\left(\mathrm{~d}, 2 \mathrm{H}, \mathrm{H}-3^{\prime \prime}, 5^{\prime \prime}, J 8.8 \mathrm{~Hz}\right) ; 6.93(\mathrm{dt}, 1 \mathrm{H}, \mathrm{H}-6$, $J 1.3$ and $7.6 \mathrm{~Hz}) ; 7.11(\mathrm{dt}, 1 \mathrm{H}, \mathrm{H}-7, J 1.3$ and $7.6 \mathrm{~Hz}) ; 7.32\left(\mathrm{~d}, 2 \mathrm{H}, \mathrm{H}-2^{\prime}, 6^{\prime}, J 8.8 \mathrm{~Hz}\right) ; 7.37\left(\mathrm{~d}, 2 \mathrm{H}, \mathrm{H}-2^{\prime \prime}, 6^{\prime \prime}\right.$, J $8.8 \mathrm{~Hz}) ; 7.42(\mathrm{~d}, 1 \mathrm{H}, \mathrm{H}-\alpha, J 16.5 \mathrm{~Hz}) ; 7.56(\mathrm{dd}, 1 \mathrm{H}, \mathrm{H}-5, J 1.3$ and $8.0 \mathrm{~Hz}) \mathrm{ppm} .{ }^{13} \mathrm{C}-\mathrm{NMR}(75.47 \mathrm{MHz}$; $\left.\mathrm{CDCl}_{3}\right): \delta 14.7\left(4^{\prime \prime}-\mathrm{OCH}_{2} \mathrm{CH}_{3}\right) ; 55.1\left(4^{\prime}-\mathrm{OCH}_{3}\right) ; 63.5\left(4^{\prime \prime}-\mathrm{OCH}_{2} \mathrm{CH}_{3}\right) ; 76.9(\mathrm{C}-2) ; 113.8\left(\mathrm{C}-3^{\prime}, 5^{\prime}\right) ; 114.6$ $\left(\mathrm{C}-3^{\prime \prime}, 5^{\prime \prime}\right) ; 116.6$ (C-8); 120.9 (C- $\left.\alpha\right) ; 121.5$ (C-6); 122.4 (C-4a); 124.8 (C-5); 127.7 (C-4); 128.1 (C-2" , , 6"); 128.7 (C-3); 129.1 (C-2', 6' ); 129.4 (C-1" ); 129.9 (C-1'); 130.0 (C-7); 131.4 (C- $\beta$ ); 152.0 (C-8a); 159.2 (C-4"'); $159.7\left(\mathrm{C}-4^{\prime}\right) \mathrm{ppm}$.

(Z)-4-Choro-2-(4-methoxyphenyl)-3-(4-nitrovinyl)-2H-chromene (10f). Yellow oil, $\eta 53 \% .{ }^{1} \mathrm{H}-\mathrm{NMR}$ $\left(300.13 \mathrm{MHz} ; \mathrm{CDCl}_{3}\right): \delta 3.74$ (s, 3H, 4-OCH $\left.)_{3}\right) ; .72(\mathrm{~s}, 1 \mathrm{H}, \mathrm{H}-2) ; 6.51$ (d, $\left.1 \mathrm{H}, \mathrm{H}-\alpha, J 12.3 \mathrm{~Hz}\right) ; 6.64(\mathrm{~d}, 1 \mathrm{H}$, $\mathrm{H}-\beta, J 12.3 \mathrm{~Hz}$ ); 6.78 (dlargo, 1H, H-8, J $8.0 \mathrm{~Hz}) ; 6.82$ (d, 2H, H-3', 5', J 8.7 Hz); 6.97 (dt, 1H, H-6, J 1.3 and 7.6 Hz); 7.18 (td, 1H, H-7, J 1.3 and $7.6 \mathrm{~Hz}) ; 7.31$ (d, 2H, H-2' '6', J $8.7 \mathrm{~Hz}) ; 7.55$ (d, 2H, H-2" , 6" , J $8.7 \mathrm{~Hz}) ; 7.61$ (d, 1H, H-5, J 8.0 Hz); $8.17\left(\mathrm{~d}, 2 \mathrm{H}, \mathrm{H}-3^{\prime \prime}, 5^{\prime \prime}\right.$, J 8.8 Hz) ppm. ${ }^{13} \mathrm{C}-\mathrm{NMR}\left(75.47 \mathrm{MHz} ; \mathrm{CDCl}_{3}\right)$ : $\delta 55.2\left(4^{\prime}-\mathrm{OCH}_{3}\right) ; 78.2(\mathrm{C}-2) ; 114.0\left(\mathrm{C}-3^{\prime}, 5^{\prime}\right) ; 116.9$ (C-8); 121.6 (C-4a); 121.8 (C-6); $124.1\left(\mathrm{C}-3^{\prime \prime}, 5^{\prime \prime}\right) ; 125.4$ (C-5); 126.5 (C-4); 127.1 (C-2" , $\left.6^{\prime \prime}\right) ; 127.2\left(\mathrm{C}-4^{\prime}\right) ; 128.2(\mathrm{C}-\alpha) ; 129.0\left(\mathrm{C}-2^{\prime}, 6^{\prime}\right) ; 129.2\left(\mathrm{C}-1^{\prime}\right) ; 129.3(\mathrm{C}-3)$;

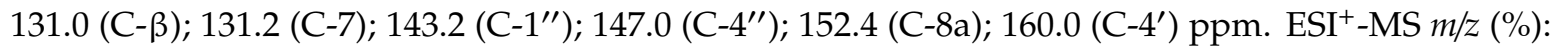
$442.1 \mathrm{Cl}^{35}[\mathrm{M}+\mathrm{Na}]^{+}(20) ; 444.1 \mathrm{Cl}^{37}[\mathrm{M}+\mathrm{Na}]^{+}(10)$. EI-HRMS $\mathrm{m} / z$ calcd for $\left(\mathrm{C}_{24} \mathrm{H}_{18} \mathrm{~N}^{35} \mathrm{ClO}_{4}\right) 419.0924$, found 419.0932. Calcd for $\left(\mathrm{C}_{24} \mathrm{H}_{18} \mathrm{~N}^{37} \mathrm{ClO}_{4}\right) 421.0895$, found 421.0895 .

(E)-4-Chloro-2-(4-methoxyphenyl)-3-(4-nitrovinyl)-2H-chromene (11f). Yellow solid, $\eta 35 \%, \mathrm{~m}$. p. $170-172{ }^{\circ} \mathrm{C} .{ }^{1} \mathrm{H}-\mathrm{NMR}\left(300.13 \mathrm{MHz} ; \mathrm{CDCl}_{3}\right): \delta 3.75\left(\mathrm{~s}, 3 \mathrm{H}, 4^{\prime}-\mathrm{OCH}_{3}\right) ; 6.27$ (s, $\left.1 \mathrm{H}, \mathrm{H}-2\right) ; 6.47$ (d, $1 \mathrm{H}, \mathrm{H}-\beta, J 16.6 \mathrm{~Hz}) ; 6.78(\mathrm{dd}, 1 \mathrm{H}, \mathrm{H}-8, J 1.3$ and $8.0 \mathrm{~Hz}) ; 6.82\left(\mathrm{~d}, 2 \mathrm{H}, \mathrm{H}-3^{\prime}, 5^{\prime}, J 8.8 \mathrm{~Hz}\right) ; 6.97(\mathrm{dt}, 1 \mathrm{H}$, H-6, J 1.3 and 7.6 Hz); $7.17(\mathrm{dt}, 1 \mathrm{H}, \mathrm{H}-7, J 1.3$ and $7.6 \mathrm{~Hz}) ; 7.31\left(\mathrm{~d}, 2 \mathrm{H}, \mathrm{H}-2^{\prime}, 6^{\prime}, J\right.$ 8 $\left.8.8 \mathrm{~Hz}\right) ; 7.55(\mathrm{~d}, 2 \mathrm{H}$, $\left.\mathrm{H}-2^{\prime \prime}, 6^{\prime \prime}, J 8.8 \mathrm{~Hz}\right) ; 7.61(\mathrm{dd}, 1 \mathrm{H}, \mathrm{H}-5, J 1.3$ and $8.0 \mathrm{~Hz}) ; 7.68(\mathrm{~d}, 1 \mathrm{H}, \mathrm{H}-\alpha, J 16.6 \mathrm{~Hz}) ; 8.17\left(\mathrm{~d}, 2 \mathrm{H}, \mathrm{H}-3^{\prime \prime}, 5^{\prime \prime}\right.$, J $8.8 \mathrm{~Hz})$ ppm. ${ }^{13} \mathrm{C}-\mathrm{NMR}\left(75.47 \mathrm{MHz} ; \mathrm{CDCl}_{3}\right): \delta 55.2\left(4^{\prime}-\mathrm{OCH}_{3}\right) ; 76.8(\mathrm{C}-2) ; 114.0\left(\mathrm{C}-3^{\prime}, 5^{\prime}\right) ; 116.9(\mathrm{C}-8)$;

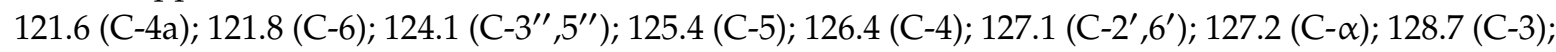
$128.9(\mathrm{C}-\beta) ; 129.0\left(\mathrm{C}-2^{\prime \prime}, 6^{\prime \prime}\right) ; 129.2\left(\mathrm{C}-1^{\prime}\right) ; 131.2(\mathrm{C}-7) ; 143.4\left(\mathrm{C}-1^{\prime \prime}\right) ; 147.0\left(\mathrm{C}-4^{\prime \prime}\right) ; 152.4(\mathrm{C}-8 \mathrm{a}) ; 160.0$ $\left(\mathrm{C}-4^{\prime}\right) \mathrm{ppm}$.

(Z)-4-Chloro-2-(4-nitrophenyl)-3-vinyl-2H-chromene (10g). Red oil, $\eta 34 \% .{ }^{1} \mathrm{H}-\mathrm{NMR}(300.13 \mathrm{MHz}$; $\left.\mathrm{CDCl}_{3}\right): \delta 5.30(\mathrm{~s}, 1 \mathrm{H}, \mathrm{H}-2) ; 6.48(\mathrm{~d}, 1 \mathrm{H}, \mathrm{H}-\alpha, J 12.2 \mathrm{~Hz}) ; 6.72(\mathrm{~d}, 1 \mathrm{H}, \mathrm{H}-\beta, J 12.2 \mathrm{~Hz}) ; 6.80(\mathrm{dd}, 1 \mathrm{H}$, H-8, J 1.3 and $8.0 \mathrm{~Hz}) ; 6.99(\mathrm{dt}, 1 \mathrm{H}, \mathrm{H}-6, J 1.3$ and $7.6 \mathrm{~Hz}) ; 7.20(\mathrm{dt}, 1 \mathrm{H}, \mathrm{H}-7, J 1.3$ and $7.6 \mathrm{~Hz}) ; 7.30(\mathrm{~d}$, 2H, H-2' , $6^{\prime}, J$ 9.0 Hz); 7.33-7.39 (m, 5H, H-2" $\left., 3^{\prime \prime}, 4^{\prime \prime}, 5^{\prime \prime}, 6^{\prime \prime}\right) ; 7.56(\mathrm{dd}, 1 \mathrm{H}, \mathrm{H}-5, J 1.3$ and $8.0 \mathrm{~Hz}) ; 8.05$ (d, 2H, H-3' ,5' , J 9.0 Hz) ppm. ${ }^{13} \mathrm{C}-\mathrm{NMR}\left(75.47 \mathrm{MHz} ; \mathrm{CDCl}_{3}\right): \delta 77.3$ (C-2); 116.2 (C-8); 121.0 (C-4a);

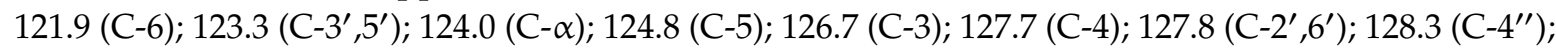
$128.4\left(\mathrm{C}-3^{\prime \prime}, 5^{\prime \prime}\right) ; 128.5\left(\mathrm{C}-2^{\prime \prime}, 6^{\prime \prime}\right) ; 130.7$ (C-7); 133.7 (C- $\left.\beta\right) ; 136.3\left(\mathrm{C}-1^{\prime \prime}\right) ; 145.8\left(\mathrm{C}-4^{\prime}\right) ; 147.5\left(\mathrm{C}-1^{\prime}\right) ; 152.1$ 
(C-8a) ppm. ESI ${ }^{+}$-MS m/z (\%): $412.1 \mathrm{Cl}^{35}[\mathrm{M}+\mathrm{Na}]^{+}(15) ; 414.3 \mathrm{Cl}^{37}[\mathrm{M}+\mathrm{Na}]^{+}$(10). EI-HRMS m/z calcd for $\left(\mathrm{C}_{23} \mathrm{H}_{16} \mathrm{~N}^{35} \mathrm{ClO}_{3}\right) 389.0819$, found 389.0817. Calcd for $\left(\mathrm{C}_{23} \mathrm{H}_{16} \mathrm{~N}^{37} \mathrm{ClO}_{3}\right)$ 391.0789; found 391.0804.

(E)-4-Chloro-2-(4-nitrophenyl)-3-vinyl-2H-chromene (11g). Orange oil, $\eta 15 \% .{ }^{1} \mathrm{H}-\mathrm{NMR}$ (300.13 MHz; $\left.\mathrm{CDCl}_{3}\right): \delta 6.38(\mathrm{~s}, 1 \mathrm{H}, \mathrm{H}-2) ; 6.48(\mathrm{~d}, 1 \mathrm{H}, \mathrm{H}-\beta, J 16.5 \mathrm{~Hz}) ; 6.84(\mathrm{dd}, 1 \mathrm{H}, \mathrm{H}-8, J 1.2$ and $8.1 \mathrm{~Hz}) ; 6.99$ (ddd, $1 \mathrm{H}, \mathrm{H}-6, J$ J 1.2, 7.0 and $8.1 \mathrm{~Hz}$ ); 7.17 (ddd, $1 \mathrm{H}, \mathrm{H}-7, J 1.2,7.0$ and $8.1 \mathrm{~Hz}$ ); 7.28-7.37 (m, 5H, H-2" , $\left.3^{\prime \prime}, 4^{\prime \prime}, 5^{\prime \prime}, 6^{\prime \prime}\right) ; 7.57$ (dlargo, 1H, H-5, J 8.1 Hz); 7.60 (d, 2H, H-2', 6' , J 9.0 Hz); 7.61 (d, 1H, $\mathrm{H}-\alpha, J 16.5 \mathrm{~Hz}) ; 8.14\left(\mathrm{~d}, 2 \mathrm{H}, \mathrm{H}-3^{\prime}, 5^{\prime}, J 9.0 \mathrm{~Hz}\right) \mathrm{ppm} .{ }^{13} \mathrm{C}-\mathrm{NMR}\left(75,47 \mathrm{MHz} ; \mathrm{CDCl}_{3}\right): \delta 75.6(\mathrm{C}-2) ; 116.7$

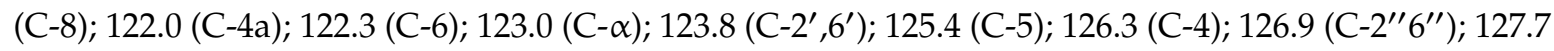
$\left(\mathrm{C}-1^{\prime \prime}\right) ; 128.7\left(\mathrm{C}-3^{\prime}, 5^{\prime}\right) ; 128.8\left(\mathrm{C}-4^{\prime \prime}\right) ; 128.9\left(\mathrm{C}-3^{\prime \prime}, 5^{\prime \prime}\right) ; 130.8$ (C-7); $132.0(\mathrm{C}-\beta) ; 136.3(\mathrm{C}-3) ; 144.9\left(\mathrm{C}-1^{\prime}\right)$; $148.0\left(\mathrm{C}-4^{\prime}\right) ; 151.8(\mathrm{C}-8 \mathrm{a}) \mathrm{ppm}$.

(Z)-4-Chloro-3-(4-ethoxyvinyl)-2-(4-nitrophenyl)-2H-chromene (10h). Yellow oil, $\eta 45 \% .{ }^{1} \mathrm{H}-\mathrm{NMR}$ (300.13 MHz; $\left.\mathrm{CDCl}_{3}\right): \delta 1.43\left(\mathrm{t}, 3 \mathrm{H}, 4^{\prime \prime}-\mathrm{OCH}_{2} \mathrm{CH}_{3}, J 7.0 \mathrm{~Hz}\right) ; 4.06\left(\mathrm{q}, 2 \mathrm{H}, 4^{\prime \prime}-\mathrm{OCH}_{2} \mathrm{CH}_{3}, J 7.0 \mathrm{~Hz}\right)$; 6.03 (s, 1H, H-2); 6.34 (d, 1H, H- $\alpha, J 12.1 \mathrm{~Hz}) ; 6.65$ (d, 1H, H- $\beta, J 12.1 \mathrm{~Hz}) ; 6.82$ (dd, 1H, H-8, J 1.3 and $8.0 \mathrm{~Hz}) ; 6.87\left(\mathrm{~d}, 2 \mathrm{H}, \mathrm{H}-3^{\prime \prime}, 5^{\prime \prime}, J 9.0 \mathrm{~Hz}\right) ; 6.99(\mathrm{dt}, 1 \mathrm{H}, \mathrm{H}-6, J 1.3$ and $7.6 \mathrm{~Hz}) ; 7.20(\mathrm{dt}, 1 \mathrm{H}, \mathrm{H}-7, J) 1.3$ and $7.6 \mathrm{~Hz}) ; 7.34\left(\mathrm{~d}, 4 \mathrm{H}, \mathrm{H}-2^{\prime}, 6^{\prime}, 2^{\prime \prime}, 6^{\prime \prime}, J 9.0 \mathrm{~Hz}\right) ; 7.55(\mathrm{dd}, 1 \mathrm{H}, \mathrm{H}-5, J 1.3$ and $8.0 \mathrm{~Hz}) ; 8.05\left(\mathrm{~d}, 2 \mathrm{H}, \mathrm{H}-3^{\prime}, 5^{\prime}\right.$, $J 9.0 \mathrm{~Hz}) \mathrm{ppm}{ }^{13} \mathrm{C}-\mathrm{NMR}\left(75.47 \mathrm{MHz} ; \mathrm{CDCl}_{3}\right): \delta 14.8\left(4^{\prime \prime}-\mathrm{OCH}_{2} \mathrm{CH}_{3}\right) ; 63.5\left(4^{\prime \prime}-\mathrm{OCH}_{2} \mathrm{CH}_{3}\right), 76.7$ (C-2);

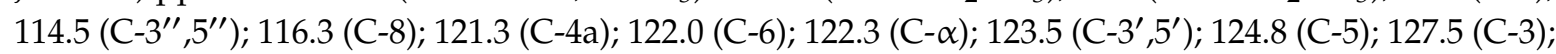
$127.9\left(\mathrm{C}-2^{\prime \prime}, 6^{\prime \prime}\right) ; 128.3$ (C-4); $128.7\left(\mathrm{C}-1^{\prime \prime}\right) ; 130.1\left(\mathrm{C}-2^{\prime}, 6^{\prime}\right) ; 130.7$ (C-7); 133.6 (C- $\left.\beta\right) ; 146.2\left(\mathrm{C}-4^{\prime}\right) ; 147.4$ (C-1'); 152.2 (C-8a); 159.1 (C-4") ppm. ESI ${ }^{+}$-MS $m / z(\%): 456.1{ }^{35} \mathrm{Cl}[\mathrm{M}+\mathrm{Na}]^{+}(45) ; 458.1{ }^{37} \mathrm{Cl}[\mathrm{M}+\mathrm{Na}]^{+}$ (15). $\mathrm{EI}^{+}$-HRMS $\mathrm{m} / \mathrm{z}$ calcd for $\left(\mathrm{C}_{25} \mathrm{H}_{20} \mathrm{NO}_{4}{ }^{35} \mathrm{Cl}\right) 433.1081$; found 433.1084 . Calcd for $\left(\mathrm{C}_{25} \mathrm{H}_{20} \mathrm{NO}_{2}{ }^{37} \mathrm{Cl}\right)$ 435.1051; found 435.1060 .

(E)-4-Chloro-3-(4-ethoxyvinyl)-2-(4-nitrophenyl)-2H-chromene (11h). Yellow oil, $\eta 24 \% .{ }^{1} \mathrm{H}-\mathrm{NMR}$ (300.13 MHz; $\left.\mathrm{CDCl}_{3}\right): \delta 1.43\left(\mathrm{t}, 3 \mathrm{H}, 4^{\prime \prime}-\mathrm{OCH}_{2} \mathrm{CH}_{3}, J 7.0 \mathrm{~Hz}\right) ; 4.06\left(\mathrm{q}, 2 \mathrm{H}, 4^{\prime \prime}-\mathrm{OCH}_{2} \mathrm{CH}_{3}, J 7.0 \mathrm{~Hz}\right) ; 6.36$ (s, $1 \mathrm{H}, \mathrm{H}-2) ; 6.42(\mathrm{~d}, 1 \mathrm{H}, \mathrm{H}-\beta, J 16.5 \mathrm{~Hz}) ; 6.83(\mathrm{dd}, 1 \mathrm{H}, \mathrm{H}-8, J 1.4$ and $\left.8.0 \mathrm{~Hz}) ; 6.87\left(\mathrm{~d}, 2 \mathrm{H}, \mathrm{H}-3^{\prime \prime}, 5^{\prime \prime}, J\right) .0 \mathrm{~Hz}\right)$; $6.97(\mathrm{dt}, 1 \mathrm{H}, \mathrm{H}-6, J 1.4$ and $7.8 \mathrm{~Hz}) ; 7.15(\mathrm{dt}, 1 \mathrm{H}, \mathrm{H}-7, J 1.4$ and $7.8 \mathrm{~Hz}) ; 7.39\left(\mathrm{~d}, 2 \mathrm{H}, \mathrm{H}-2^{\prime \prime}, 6^{\prime \prime}, J 9.0 \mathrm{~Hz}\right)$; $7.46(\mathrm{~d}, 1 \mathrm{H}, \mathrm{H}-\alpha, J 16.5 \mathrm{~Hz}) ; 7.56(\mathrm{~d}, 1 \mathrm{H}, \mathrm{H}-5, J 1.4$ and $8.0 \mathrm{~Hz}) ; 7.60\left(\mathrm{~d}, 2 \mathrm{H}, \mathrm{H}-2^{\prime}, 6^{\prime}, J\right.$, $\left.9.0 \mathrm{~Hz}\right) ; 8.13(\mathrm{~d}$, 2H, H-3', 5', J $9.0 \mathrm{~Hz}) \mathrm{ppm} .{ }^{13} \mathrm{C}-\mathrm{NMR}\left(75.47 \mathrm{MHz} ; \mathrm{CDCl}_{3}\right): \delta 14.6\left(4^{\prime \prime}-\mathrm{OCH}_{2} \mathrm{CH}_{3}\right) ; 63.4\left(4^{\prime \prime}-\mathrm{OCH}_{2} \mathrm{CH}_{3}\right)$,

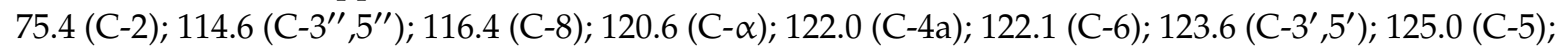
126.2 (C-4); $126.4\left(\mathrm{C}-1^{\prime \prime}\right) ; 128.1\left(\mathrm{C}-2^{\prime}, 6^{\prime}\right) ; 128.5\left(\mathrm{C}-2^{\prime \prime}, 6^{\prime \prime}\right) ; 128.7$ (C-3); 130.2 (C-7); 131.5 (C- $\left.\beta\right) ; 144.8$ $\left(\mathrm{C}-1^{\prime}\right) ; 147.8$ (C-4'); 151.4 (C-8a); $159.4\left(\mathrm{C}-4^{\prime \prime}\right)$ ppm.

(Z)-4-Chloro-2-(4-nitrophenyl)-3-(4-nitrovinyl)-2H-chromene (10i). Orange solid, $\eta 52 \%$, m. p. 163-165 ${ }^{\circ} \mathrm{C} .{ }^{1} \mathrm{H}-\mathrm{NMR}\left(300.13 \mathrm{MHz} ; \mathrm{CDCl}_{3}\right): \delta 5.85$ (s, $\left.1 \mathrm{H}, \mathrm{H}-2\right) ; 6.63(\mathrm{~d}, 1 \mathrm{H}, \mathrm{H}-\alpha, J 12.2 \mathrm{~Hz}) ; 6.74(\mathrm{~d}, 1 \mathrm{H}$, $\mathrm{H}-\beta, J 12.2 \mathrm{~Hz}$ ); 6.84 (dd, 1H, H-8, J 1.3 and $8.0 \mathrm{~Hz}$ ); 7.01 (ddd, 1H, H-6, J 1.3, 7.3 and $8.0 \mathrm{~Hz}$ ); 7.24 (ddd, $1 \mathrm{H}, \mathrm{H}-7, J 1.3,7.3$ and $8.0 \mathrm{~Hz}) ; 7.36\left(\mathrm{~d}, 2 \mathrm{H}, \mathrm{H}-2^{\prime}, 6^{\prime}, J 8.8 \mathrm{~Hz}\right) ; 7.56(\mathrm{dd}, 1 \mathrm{H}, \mathrm{H}-5, J 1.3$ and $8.0 \mathrm{~Hz}) ; 7.58(\mathrm{~d}$,

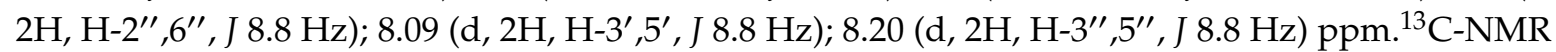
(75.47 MHz; $\left.\mathrm{CDCl}_{3}\right)$ : $\delta 77.0$ (C-2); 116.6 (C-8); 120.8 (C-4a); 122.4 (C-6); $123.7\left(\mathrm{C}-3^{\prime \prime}, 5^{\prime \prime}\right) ; 124.0\left(\mathrm{C}-3^{\prime}, 5^{\prime}\right)$;

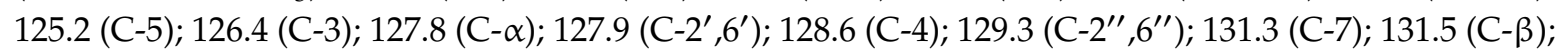
$142.8\left(\mathrm{C}-1^{\prime \prime}\right) ; 145.3\left(\mathrm{C}-1^{\prime}\right) ; 147.3\left(\mathrm{C}-4^{\prime \prime}\right) ; 147.9\left(\mathrm{C}-4^{\prime}\right) ; 152.2$ (C-8a) ppm. $\mathrm{ESI}^{+}-\mathrm{MS} \mathrm{m} / \mathrm{z}(\%): 457.1 \mathrm{Cl}^{35}$ $[\mathrm{M}+\mathrm{Na}]^{+}(80) ; 459.1 \mathrm{Cl}^{37}[\mathrm{M}+\mathrm{Na}]^{+}(15)$. EI-HRMS $\mathrm{m} / z$ calcd for $\left(\mathrm{C}_{23} \mathrm{H}_{15} \mathrm{~N}_{2}{ }^{35} \mathrm{ClO}_{5}\right) 434.0669$, found 434.0668. Calcd for $\left(\mathrm{C}_{23} \mathrm{H}_{15} \mathrm{~N}_{2}{ }^{37} \mathrm{ClO}_{5}\right) 436.0640$, found 436.0647 .

(E)-4-Chloro-2-(4-nitrophenyl)-3-(4-nitrovinyl)-2H-chromene (11i). Orange solid, $\rceil 27 \%$, m. p. 180-182 ${ }^{\circ} \mathrm{C} .{ }^{1} \mathrm{H}-\mathrm{NMR}\left(300.13 \mathrm{MHz} ; \mathrm{CDCl}_{3}\right): \delta 6.38(\mathrm{~s}, 1 \mathrm{H}, \mathrm{H}-2) ; 6.49(\mathrm{~d}, 1 \mathrm{H}, \mathrm{H}-\beta, J 16.5 \mathrm{~Hz}) ; 6.85(\mathrm{dd}, 1 \mathrm{H}$, $\mathrm{H}-8, J 1.3$ and $8.0 \mathrm{~Hz}) ; 7.01(\mathrm{ddd}, 1 \mathrm{H}, \mathrm{H}-6, J 1.3,7.6$ and $8.0 \mathrm{~Hz}) ; 7.21$ (ddd, $1 \mathrm{H}, \mathrm{H}-7, J$ J 1.3, 7.6 and $8.0 \mathrm{~Hz}$ );

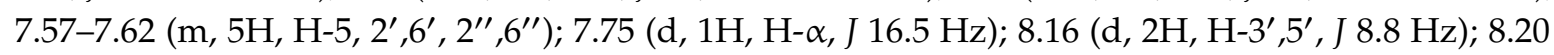
(d, 2H, H-3" , , 5" , J $8.8 \mathrm{~Hz}$ ) ppm. ${ }^{13} \mathrm{C}-\mathrm{NMR}$ : $\delta 75.4$ (C-2); 116.9 (C-8); 121.6 (C-4a); 122.5 (C-6); 123.9 $\left(\mathrm{C}-3^{\prime \prime}, 5^{\prime \prime}\right) ; 124.2\left(\mathrm{C}-3^{\prime}, 5^{\prime}\right) ; 125.5(\mathrm{C}-4) ; 125.8(\mathrm{C}-5) ; 127.1(\mathrm{C}-\alpha) ; 127.2\left(\mathrm{C}-2^{\prime \prime}, 6^{\prime \prime}\right) ; 128.6\left(\mathrm{C}-2^{\prime}, 6^{\prime}\right) ; 129.1$ (C- $\beta) ; 130.6$ (C-3); 131.6 (C-7); 142.6 (C-1" ); 144.3 (C-1'); 147.2 (C-4" ); 148.1 (C-4'); 152.0 (C-8a) ppm. 
3.6. General Procedure for the Synthesis of (Z- and E)-2-aryl-4-chloro-3-styrylquinoline-1(2H)-carbaldehydes $12 \mathbf{a}-\mathbf{i} / \mathbf{1 3} \mathbf{a}-\mathbf{i}$

The appropriate phosphonium halide salt $(8 \mathbf{a}-\mathbf{c}, 0.20 \mathrm{mmol})$ in dry THF $(20 \mathrm{~mL})$ and sodium hydride $(6 \mathrm{mg}, 0.20 \mathrm{mmol})$ were placed in a previously dried flask. The mixture was heated at $60^{\circ} \mathrm{C}$ for 15,7 and $3 \mathrm{~min}$ to prepare the ylide $\mathbf{9 a}, \mathbf{9 b}$ and $\mathbf{9 c}$, respectively. After these periods, the formylated derivative $\mathbf{2} \mathbf{d}-\mathbf{f}$ was added $(0.07 \mathrm{mmol})$. The reaction mixture was refluxed or maintained at room temperature for the appropriate time depending of each formylated derivative (see Table 2). At the end, the reaction mixture was poured into water $(40 \mathrm{~mL})$ and ice $(50 \mathrm{~g})$, the $\mathrm{pH}$ adjusted to 3 with dilute hydrochloric acid. The reaction mixture was extracted with ethyl acetate $(3 \times 20 \mathrm{~mL})$, the solvent was dried using anhydrous sodium sulphate and evaporated to dryness on the evaporator. The residue was purified by thin layer chromatography using mixtures of dichloromethane/hexane as eluent.

(E)-4-Chloro-2-phenyl-3-styrylquinoline-1(2H)-carbaldehyde (12a). Yellow solid, $\eta 44 \%$ (11 mg), m.p. $72-74{ }^{\circ} \mathrm{C} .{ }^{1} \mathrm{H}-\mathrm{NMR}\left(300.13 \mathrm{MHz} ; \mathrm{CDCl}_{3}\right): \delta 6.80$ (d, 1H, H- $\left.3, J 16.4 \mathrm{~Hz}\right) ; 6.97$ (s, 1H, H-2); 7.02-7.05 (m, 1H, H-8); 7.20-7.35 (m, 10H, H-6,7, $\left.3^{\prime}, 4^{\prime}, 5^{\prime}, 2^{\prime \prime}, 3^{\prime \prime}, 4^{\prime \prime}, 5^{\prime \prime}, 6^{\prime \prime}\right) ; 7.46$ (m, 2H, H-2' , 6' ); 7.57 (d, $1 \mathrm{H}, \mathrm{H}-\alpha$, J 16.4 Hz); 7.81-7.84 (m, 1H, H-5); 8.68 (s, 1H, 1-NCHO) ppm. ${ }^{13} \mathrm{C}-\mathrm{NMR}\left(75.47 \mathrm{MHz} ; \mathrm{CDCl}_{3}\right): \delta 52.2$ (C-2); 118.2 (C-8); 123.1 (C- $\beta$ ); 126.0 (C-5); 126.2 (C-6); 126.5 (C-4a); 127.0 (C-4); 127.1 (C-2' ,6'); 127.5 $\left(\mathrm{C}-2^{\prime \prime}, 6^{\prime \prime}\right) ; 128.3\left(\mathrm{C}-4^{\prime}\right) ; 128.6\left(\mathrm{C}-4^{\prime \prime}\right) ; 128.8\left(\mathrm{C}-3^{\prime}, 5^{\prime}, 3^{\prime \prime}, 5^{\prime \prime}\right) ; 129.6(\mathrm{C}-7) ; 131.4\left(\mathrm{C}-1^{\prime}\right) ; 133.6(\mathrm{C}-\alpha) ; 134.1$ (C-3); 136.5 (C-1" ); 137.2 (C-8a); 161.0 (1-NCHO) ppm. MS (ESI $\left.{ }^{+}\right) \mathrm{m} / z(\%): 394.1{ }^{35} \mathrm{Cl}[\mathrm{M}+\mathrm{Na}]^{+}(100)$, $396.1{ }^{37} \mathrm{Cl}[\mathrm{M}+\mathrm{Na}]^{+}$(38). MS $\left(\mathrm{ESI}^{+}\right)$calcd for $\left(\mathrm{C}_{24} \mathrm{H}_{18}{ }^{35} \mathrm{ClNO}\right) 371.1077$; found 371.1078. Calcd for $\left(\mathrm{C}_{24} \mathrm{H}_{18}{ }^{37} \mathrm{ClNO}\right) 373.1047$; found 372.1048.

(E)-4-Chloro-3-(4-nitrostyryl)-2-phenylquinoline-1(2H)-carbaldehyde (12c). Orange solid, $\eta 40 \%$ (12 mg), m.p. $243.5-245.3{ }^{\circ} \mathrm{C} .{ }^{1} \mathrm{H}-\mathrm{NMR}\left(300.13 \mathrm{MHz} ; \mathrm{CDCl}_{3}\right): \delta 6.81$ (d, $\left.1 \mathrm{H}, \mathrm{H}-\beta, J 16.4 \mathrm{~Hz}\right) ; 6.96(\mathrm{~s}, 1 \mathrm{H}$, H-2); 7.04-7.07 (m, 1H, H-8); 7.23-7.32 (m, 7H, H-6,7, 2' , $\left.3^{\prime}, 4^{\prime}, 5^{\prime}, 6^{\prime}\right) ; 7.59$ (d, 2H, H-2' ,6' , J 8.8 Hz); 7.71 (d, $1 \mathrm{H}, \mathrm{H}-\alpha, J 16.4 \mathrm{~Hz}) ; 7.85-7.88$ (m, 1H, H-5); 8.19 (d, 2H, H-3" , 5" , J 8.8 Hz); 8.67 (s, 1H, 1-NCHO) ppm. ${ }^{13} \mathrm{C}-\mathrm{NMR}\left(75.47 \mathrm{MHz} ; \mathrm{CDCl}_{3}\right)$ : $\delta 52.1$ (C-2); 118.3 (C-8); 124.1 (C-3" , , $\left.{ }^{\prime \prime}\right) ; 125.9$ (C-4a); 126.2 (C-6); 126.7 (C-5); $127.2(\mathrm{C}-\beta) ; 127.3(\mathrm{C}-4) ; 127.4\left(\mathrm{C}-2^{\prime \prime}{ }^{\prime}, 6^{\prime \prime}, 2^{\prime}, 6^{\prime}\right) ; 128.6\left(\mathrm{C}-4^{\prime}\right) ; 128.7\left(\mathrm{C}-3^{\prime}, 5^{\prime}\right) ; 129.1$ (C-3); $130.4(\mathrm{C}-7)$;

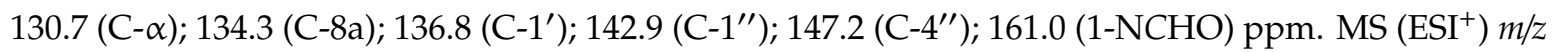
(\%): $417.9{ }^{35} \mathrm{Cl}[\mathrm{M}+\mathrm{H}]^{+}(100), 419.9{ }^{37} \mathrm{Cl}[\mathrm{M}+\mathrm{H}]^{+}$(3). $\mathrm{MS}\left(\mathrm{ESI}^{+}\right)$calcd for $\left(\mathrm{C}_{24} \mathrm{H}_{17}{ }^{35} \mathrm{ClN}_{2} \mathrm{O}_{3}\right) 416.0928$; found 416.0929. Calcd for $\left(\mathrm{C}_{24} \mathrm{H}_{17}{ }^{35} \mathrm{ClN}_{2} \mathrm{O}_{3}\right) 418.0898$; found 418.0100

(E)-4-Chloro-2-(4-methoxyphenyl)-3-styrylquinoline-1(2H)-carbaldehyde (12d). White solid, $\eta$ 46\% (13 mg), m.p. 173-175 ${ }^{\circ} \mathrm{C} .{ }^{1} \mathrm{H}-\mathrm{NMR}\left(300.13 \mathrm{MHz} ; \mathrm{CDCl}_{3}\right): \delta 3.72\left(\mathrm{~s}, 3 \mathrm{H}, 4^{\prime}-\mathrm{OCH}_{3}\right) ; 6.74(\mathrm{~d}, 2 \mathrm{H}$, H-3' , 5' , J $8.8 \mathrm{~Hz}) ; 6.79$ (d, 1H, H- $\beta, J 16.4 \mathrm{~Hz}) ; 6.91$ (s, 1H, H-2); 7.01-7.04 (m, 1H, H-8); 7.21 (d, 2H,

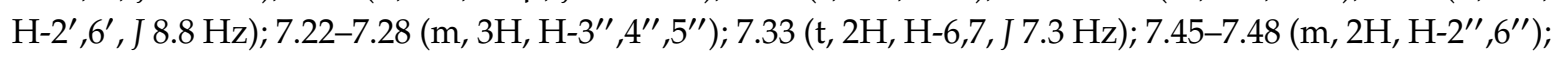
$7.54(\mathrm{~d}, 1 \mathrm{H}, \mathrm{H}-\alpha, J 16.4 \mathrm{~Hz}) ; 7.80-7.84(\mathrm{~m}, 1 \mathrm{H}, \mathrm{H}-5) ; 8.67$ (s, 1H, 1-NCHO) ppm. ${ }^{13} \mathrm{C}-\mathrm{NMR}(75.47 \mathrm{MHz}$; $\left.\mathrm{CDCl}_{3}\right): \delta 51.9(\mathrm{C}-2) ; 55.2\left(4^{\prime}-\mathrm{OCH}_{3}\right) ; 114.1\left(\mathrm{C}-3^{\prime}, 5^{\prime}\right) ; 118.2(\mathrm{C}-8) ; 123.0(\mathrm{C}-\beta) ; 125.9(\mathrm{C}-6) ; 126.2(\mathrm{C}-5)$;

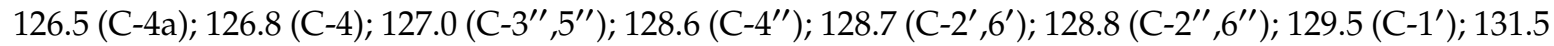

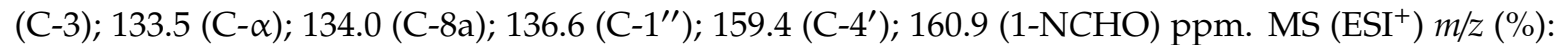
$424.1{ }^{35} \mathrm{Cl}[\mathrm{M}+\mathrm{Na}]^{+}(100) ; 426.1{ }^{37} \mathrm{Cl}[\mathrm{M}+\mathrm{Na}]^{+}(37) . \mathrm{MS}\left(\mathrm{ESI}^{+}\right)$calcd for $\left(\mathrm{C}_{25} \mathrm{H}_{21}{ }^{35} \mathrm{ClNO}_{2}\right) 402.1255$; found 402.1253. Calcd for $\left(\mathrm{C}_{25} \mathrm{H}_{21}{ }^{37} \mathrm{ClNO}_{2}\right)$ 404.1255; found 402.1254.

(E)-4-Chloro-3-(4-ethoxystyryl)-2-(4-methoxyphenyl)quinoline-1(2H)-carbaldehyde (12e). Yellow solid, $\eta 27 \%$ (8 mg), m.p. $234-236{ }^{\circ} \mathrm{C} .{ }^{1} \mathrm{H}-\mathrm{NMR}\left(300.13 \mathrm{MHz} ; \mathrm{CDCl}_{3}\right): \delta 1.41$ (t, 3H, $4^{\prime \prime}-\mathrm{OCH}_{2} \mathrm{CH}_{3}$,

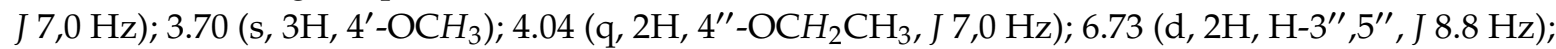
6.74 (d, 1H, H- $\beta, J 16.2 \mathrm{~Hz}) ; 6.85$ (d, 2H, H-3' ,5' , J $8.8 \mathrm{~Hz}) ; 6.89$ (s, 1H, H-2); $7.02-7.03$ (m, 1H, H-8); 7.20 (d, 2H, H-2' ,6' , J $8.8 \mathrm{~Hz}$ ); $7.23-7.26$ (m, 2H, H-6,7); 7.39 (d, 2H, H-2" ,6" $\left.{ }^{\prime \prime}, J 8.8 \mathrm{~Hz}\right) ; 7.40$ (d, $1 \mathrm{H}$, $\mathrm{H}-\alpha, J 16.2 \mathrm{~Hz}) ; 7.79-7.82(\mathrm{~m}, 1 \mathrm{H}, \mathrm{H}-5) ; 8.66$ (s, 1H, 1-NCHO) ppm. ${ }^{13} \mathrm{C}-\mathrm{NMR}\left(75.47 \mathrm{MHz} ; \mathrm{CDCl}_{3}\right): \delta$ $14.8\left(4^{\prime \prime}-\mathrm{OCH}_{2} \mathrm{CH}_{3}\right) ; 51.8(\mathrm{C}-2) ; 55.1\left(4^{\prime}-\mathrm{OCH}_{3}\right) ; 63.5\left(4^{\prime \prime}-\mathrm{OCH}_{2} \mathrm{CH}_{3}\right) ; 114.0\left(\mathrm{C}-3^{\prime \prime}, 5^{\prime \prime}\right) ; 114.7\left(\mathrm{C}-3^{\prime}, 5^{\prime}\right)$; 118.1 (C-8); 120.8 (C- $\alpha) ; 125.6$ (C-1"); 125.9 (C-5); 126.0 (C-6); 126.6 (C-4a); $128.4\left(\mathrm{C}-2^{\prime \prime}{ }^{\prime \prime} 6^{\prime \prime}\right) ; 128.8$ $\left(\mathrm{C}-2^{\prime}, 6^{\prime}\right) ; 129.2$ (C-7); 129.5 (C-1') 131.9 (C-3); 133.2 (C- $\left.\beta, 8 \mathrm{a}\right) ; 133.9$ (C-4); 159.3 (C-4'); $159.5\left(\mathrm{C}-4^{\prime \prime}\right) ; 161.0$ (1-NCHO) ppm. MS (ESI $\left.\left.{ }^{+}\right) \mathrm{m} / z(\%): 445.9{ }^{35} \mathrm{Cl}[\mathrm{M}+\mathrm{H}]^{+}(100) ; 447.9{ }^{37} \mathrm{Cl}[\mathrm{M}+\mathrm{H}]^{+}(13) \cdot \mathrm{MS}_{(\mathrm{ESI}}{ }^{+}\right)$ 
$m / z$ calcd for $\left(\mathrm{C}_{27} \mathrm{H}_{25}{ }^{35} \mathrm{ClNO}_{3}\right) 446.1516$; found 446.1517. Calcd for $\left(\mathrm{C}_{27} \mathrm{H}_{25}{ }^{37} \mathrm{ClNO}_{3}\right)$ 448.1516; found 446.1519 .

(E)-4-Chloro-2-(4-methoxyphenyl)-3-(4-nitrostyryl)quinoline-1 $(2 \mathrm{H})$-carbaldehyde (12f). Yellow solid, $\eta 53 \%$ (17 mg), m.p. $209-21{ }^{\circ} \mathrm{C} .{ }^{1} \mathrm{H}-\mathrm{NMR}\left(300.13 \mathrm{MHz} ; \mathrm{CDCl}_{3}\right): \delta 3.72\left(4^{\prime}-\mathrm{OCH}_{3}\right) ; 6.76(\mathrm{~d}, 2 \mathrm{H}$, H-3' , $\left.5^{\prime}, J 8.7 \mathrm{~Hz}\right) ; 6.80$ (d, 1H, H- $\left.\beta, J 16.1 \mathrm{~Hz}\right) ; 6.90$ (s, 1H, H-2); 7.04-7.07 (m, 1H, H-8); 7.20 (d, 2H, H-2', 6' , J 8.7 Hz); 7.29-7.32 (m, 2H, H-6,7); 7.58 (d, 2H, H-2' , 6" , J 8.7 Hz); 7.69 (d, 1H, H- $\alpha, J 16.1 \mathrm{~Hz})$; 7.84-7.88 (m, 1H, H-5); 8.18 (d, 2H, H-3", $5^{\prime \prime}$, J $\left.8.7 \mathrm{~Hz}\right) ; 8.67$ (s, 1H, 1-NCHO) ppm. ${ }^{13} \mathrm{C}-\mathrm{NMR}(75.47 \mathrm{MHz}$; $\left.\mathrm{CDCl}_{3}\right): \delta 51.8(\mathrm{C}-2) ; 55.2\left(\mathrm{C}-4^{\prime}\right) ; 114.2\left(\mathrm{C}-3^{\prime}, 5^{\prime}\right) ; 118.3(\mathrm{C}-8) ; 124.1\left(\mathrm{C}-3^{\prime \prime}, 5^{\prime \prime}\right) ; 126.0(\mathrm{C}-4 \mathrm{a}) ; 126.1$ (C-6); 126.6 (C-5); 127.2 (C- $\beta) ; 127.4\left(\mathrm{C}-2^{\prime}, 6^{\prime}\right) ; 128.7\left(\mathrm{C}-2^{\prime \prime}, 6^{\prime \prime}\right) ; 128.9$ (C-1'); 129.6 (C-4); 130.4 (C-7); 130.6 $(\mathrm{C}-\alpha) ; 130.8(\mathrm{C}-3) ; 134.3(\mathrm{C}-8 \mathrm{a}) ; 143.0\left(\mathrm{C}-1^{\prime \prime}\right) ; 147.2\left(\mathrm{C}-4^{\prime \prime}\right) ; 159.6\left(\mathrm{C}-4^{\prime}\right) ; 160.9$ (1-NCHO) ppm. MS (ESI $\left.{ }^{+}\right)$ $m / z(\%): 469.9{ }^{35} \mathrm{Cl}[\mathrm{M}+\mathrm{Na}]^{+}(100) ; 471.9{ }^{37} \mathrm{Cl}[\mathrm{M}+\mathrm{Na}]^{+}(2) . \mathrm{MS}\left(\mathrm{ESI}^{+}\right)$calcd for $\left(\mathrm{C}_{25} \mathrm{H}_{20}{ }^{35} \mathrm{ClN}_{2} \mathrm{O}_{4}\right)$ 447.1106; found 447.1110. Calcd for $\left(\mathrm{C}_{25} \mathrm{H}_{20}{ }^{37} \mathrm{ClN}_{2} \mathrm{O}_{4}\right)$ 449.1106; found 448.1109.

(E)-4-Chloro-2-(4-nitrophenyl)-3-styrylquinoline-1(2H)-carbaldehyde (12g). Orange solid, $\eta 30 \%$ (9 mg), m.p. 179.9-180.7 ${ }^{\circ} \mathrm{C} .{ }^{1} \mathrm{H}-\mathrm{NMR}\left(300.13 \mathrm{MHz} ; \mathrm{CDCl}_{3}\right): \delta 6.74(\mathrm{~d}, 1 \mathrm{H}, \mathrm{H}-\beta, J 16.4 \mathrm{~Hz}) ; 7.04(\mathrm{~s}, 1 \mathrm{H}$,

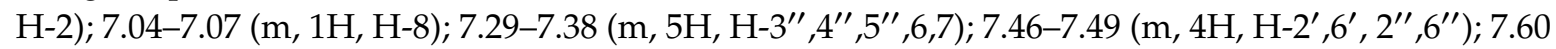
(d, 1H, H- $\alpha, J 16.4 \mathrm{~Hz}) ; 7.83-7.87$ (m, 1H, H-5); 8.09 (d, 2H, H-3', 5' , J 8.8 Hz); 8.71 (s, 1H, 1-NCHO) ppm. ${ }^{13} \mathrm{C}-\mathrm{NMR}\left(75.47 \mathrm{MHz}\right.$ C CDCl 3 ): $\delta 51.3$ (C-2); 118.2 (C-8); 122.8 (C- $\beta$ ); 124.1 (C-3', $\left.5^{\prime}\right) ; 126.1$ (C-4a); 126.5

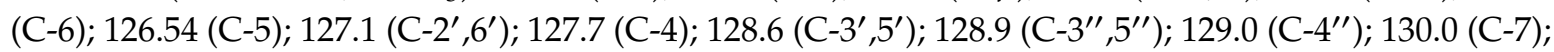
130.2 (C-8a); 133.5 (C-3); 133.7 (C- $\alpha) ; 136.1\left(\mathrm{C}-1^{\prime}\right) ; 144.3\left(\mathrm{C}-1^{\prime}\right) ; 147.8$ (C-4'); 161.0 (1-NCHO) ppm. MS $\left(\mathrm{ESI}^{+}\right) \mathrm{m} / \mathrm{z}(\%): 417.1^{35} \mathrm{Cl}[\mathrm{M}+\mathrm{H}]^{+}(30) ; 419.1{ }^{37} \mathrm{Cl}[\mathrm{M}+\mathrm{H}]^{+}(2)$. MS $\left(\mathrm{ESI}^{+}\right)$calcd for $\left(\mathrm{C}_{24} \mathrm{H}_{18}{ }^{35} \mathrm{ClN}_{2} \mathrm{O}_{3}\right)$ 417.0996; found 417.0999. Calcd for $\left(\mathrm{C}_{24} \mathrm{H}_{18}{ }^{37} \mathrm{ClN}_{2} \mathrm{O}_{3}\right)$ 419.0996; found 417.0997.

(E)-4-Chloro-3-(4-ethoxystyryl)-2-(4-nitrophenyl)quinoline-1(2H)-carbaldehyde (12h). Yellow oil, $\eta 24 \% .{ }^{1} \mathrm{H}-\mathrm{NMR}\left(300.13 \mathrm{MHz} ; \mathrm{CDCl}_{3}\right): \delta 1.42\left(\mathrm{t}, 3 \mathrm{H}, 4^{\prime \prime}-\mathrm{OCH}_{2} \mathrm{CH}_{3}, J 7.0 \mathrm{~Hz}\right) ; 4.04\left(\mathrm{q}, 2 \mathrm{H}, 4^{\prime \prime}-\mathrm{OCH}_{2} \mathrm{CH}_{3}\right.$, J 7.0); 6.69 (d, $1 \mathrm{H}, \mathrm{H}-\beta, J 16.4 \mathrm{~Hz}) ; 6.86\left(\mathrm{~d}, 2 \mathrm{H}, \mathrm{H}-3^{\prime \prime}, 5^{\prime \prime}\right.$, J $\left.8.7 \mathrm{~Hz}\right) ; 7.01$ (s, $\left.1 \mathrm{H}, \mathrm{H}-2\right) ; 7.03-7.06$ (m, $1 \mathrm{H}$, H-8); 7.27-7.31 (m, 2H, H-6,7); 7.40 (d, 2H, H-2" , 6" , J 8.7 Hz); 7.47 (d, 2H, H-2', 6' , J 8.7 Hz); 7.47 (d, $1 \mathrm{H}, \mathrm{H}-\alpha, J 16.4 \mathrm{~Hz}) ; 7.81-7.84(\mathrm{~m}, 1 \mathrm{H}, \mathrm{H}-5) ; 8.08$ (d, 2H, H-3',5', J 8.7 Hz); 8.70 (s, 1H, 1-NCHO) ppm. ${ }^{13} \mathrm{C}-\mathrm{NMR}\left(75.47 \mathrm{MHz} ; \mathrm{CDCl}_{3}\right): \delta 14.8\left(4^{\prime \prime}-\mathrm{OCH}_{2} \mathrm{CH}_{3}\right) ; 51.3(\mathrm{C}-2) ; 63.6\left(4^{\prime \prime}-\mathrm{OCH}_{2} \mathrm{CH}_{3}\right) ; 114.8\left(\mathrm{C}-3^{\prime \prime}, 5^{\prime \prime}\right)$;

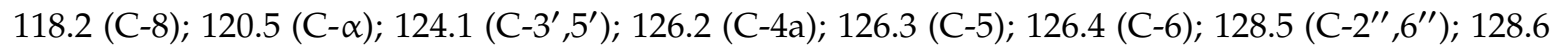
$\left(\mathrm{C}-2^{\prime}, 6^{\prime}\right) ; 128.7\left(\mathrm{C}-1^{\prime \prime}\right) ; 129.7$ (C-7); 130.5 (C-3); 133.3 (C- $\beta$ ); 133.5 (C-4); $144.4\left(\mathrm{C}-1^{\prime}\right) ; 147.7$ (C-4'); 159.8 $\left(\mathrm{C}-4^{\prime \prime}\right) ; 161.1(1-\mathrm{NCHO}) \mathrm{ppm}$. MS (ESI $\left.{ }^{+}\right) \mathrm{m} / z(\%): 461 .{ }^{35} \mathrm{Cl}[\mathrm{M}+\mathrm{H}]^{+}(50) ; 463.1{ }^{37} \mathrm{Cl}[\mathrm{M}+\mathrm{H}]^{+}$(3).MS $\left(\mathrm{ESI}^{+}\right) \mathrm{m} / z$ calcd for $\left(\mathrm{C}_{26} \mathrm{H}_{22}{ }^{35} \mathrm{ClN}_{2} \mathrm{O}_{4}\right) 461.1263$; found 461.1261. Calcd for $\left(\mathrm{C}_{26} \mathrm{H}_{22}{ }^{37} \mathrm{ClN}_{2} \mathrm{O}_{4}\right) 463.1263$; found 463.1263 .

\subsection{Theoretical Studies}

Theoretic studies were performed get an insight into the selectivity of the Wittig reaction with quinoline-3-carbaldehydes $\mathbf{2 d}-\mathbf{f}$. All relevant structures were optimized using density functional theory (DFT). Gaussian 09 et al. [33] was used for all single-point calculations employing the standard fine grid and a B3LYP/6-31G(d) [34-36] level of theory. This is in agreement with the literature that suggests that larger basis sets do not cause significant changes in the relative energies of the two diastereomeric transition states, here in study. The use of THF as solvent was simulated using a standard PCM model. Vibrational frequency calculations were performed to ensure the optimized structures represented the local minima. All transition states here shown contained a single negative frequency, and the corresponding eigenvector was verified to correspond to the expected reaction coordinate. IRC calculations were also performed and lead to the formation of the next intermediate.

\section{Conclusions}

This work establishes that 2-aryl-4-chloro-2H-chromene-3-carbaldehydes are useful building blocks and afforded several new derivatives in good yields. Interesting heterocycles, such as $3 \mathrm{H}$-chromeno quinolines, were obtained, and it also demonstrated that they could be used in Wittig reactions to get new ( $\mathrm{Z}$ and $\mathrm{E}$ )-2-aryl-4-chloro-3-styryl-2H-chromenes. It was evident that 2-aryl-4-chloro- 
1,2-dihydroquinoline-1,3-dicarbaldehydes are less reactive. However, it was also possible to use them in Wittig reactions, where they showed high selectivity towards the (E)-2-aryl-4-chloro3-styrylquinoline-1(2H)-carbaldehydes. This unique selectivity was explained through density functional theory analysis.

Author Contributions: Investigation, D.H.A.R., E.J.F.B., V.F.B.; conceptualization, D.C.G.A.P. and A.M.S.S.; supervision, D.C.G.A.P. and A.M.S.S.; validation, D.C.G.A.P. and A.M.S.S.; formal analysis, D.H.A.R., V.F.B.; D.C.G.A.P. and A.M.S.S.; writing-original draft preparation, D.H.A.R., V.F.B.; D.C.G.A.P.; writing-review and editing, D.C.G.A.P. and A.M.S.S. All authors have read and agreed to the published version of the manuscript.

Funding: This research received no external funding.

Acknowledgments: Thanks are due to the University of Aveiro and FCT/MCT for the financial support for the QOPNA research Unit (UID/QUI/00062/2019) and the LAQV-REQUIMTE (UIDB/50006/2020) througH-National funds and, where applicable, co-financed by the FEDER, within the PT2020 Partnership Agreement, and to the Portuguese NMR Network. D.H.A.R. and V.F.B. also thanks FCT for their PhD grant (SFRH/BD/68991/2010) and $(\mathrm{PD} / \mathrm{BD} / 135099 / 2017)$, respectively.

Conflicts of Interest: The authors declare no conflict of interest.

\section{References}

1. Murthy, Y.L.N.; Suhasini, K.P.; Pathania, A.S.; Bhushan, S.; Sastry, Y.N. Synthesis, structure activity relationship and mode of action of 3-substitutedphenyl-1-(2,2,8,8-tetramethyl-3,4,9,10-tetrahydro- $2 \mathrm{H}$, $8 H$-pyrano[2,3-f]chromen-6-yl)-propenones as novel anticancer agents in human leukaemia HL-60 cells. Eur. J. Med. Chem. 2013, 62, 545-555. [CrossRef] [PubMed]

2. Joshi, P.; Vishwakarma, R.A.; Bharate, S.B. Natural alkaloids as P-gp inhibitors for multidrug resistance reversal in cancer. Eur. J. Med. Chem. 2017, 138, 273-292. [CrossRef] [PubMed]

3. Conti, C.; Desideri, N. New 4H-chromen-4-one and $2 H$-chromene derivatives as anti-picornavirus capsid-binders. Bioorg. Med. Chem. 2010, 18, 6480-6488. [CrossRef] [PubMed]

4. Zheng, P.; Somersan-Karakaya, S.; Lu, S.; Roberts, J.; Pingle, M.; Warrier, T.; Little, D.; Guo, X.; Brickner, S.J.; Nathan, C.F.; et al. Synthetic calanolides with bactericidal activity against replicating and nonreplicating mycobacterium tuberculosis. J. Med. Chem. 2014, 57, 3755-3772. [CrossRef] [PubMed]

5. Felicetti, T.; Cannalire, R.; Nizi, M.G.; Tabarrini, O.; Massari, S.; Barreca, M.L.; Manfroni, G.; Schindler, B.D.; Cecchetti, V.; Kaatz, G.W.; et al. Studies on 2-phenylquinoline Staphylococcus aureus NorA efflux pump inhibitors: New insights on the C-6 position. Eur. J. Med. Chem. 2018, 155, 428-433. [CrossRef]

6. Najafi, Z.; Saeedi, M.; Mahdavi, M.; Sabourian, R.; Khanavi, M.; Tehrani, M.B.; Moghadam, F.H.; Edraki, N.; Karimpor-Razkenari, E.; Sharifzadeh, M.; et al. Design and synthesis of novel anti-Alzheimer's agents: Acridine-chromenone and quinoline-chromenone hybrids. Bioorg. Chem. 2016, 67, 84-94. [CrossRef]

7. Balabani, A.; Hadjipavlou-Litina, D.J.; Litinas, K.E.; Mainou, M.; Tsironi, C.-C.; Vronteli, A. Synthesis and biological evaluation of (2,5-dihydro- $1 H$-pyrrol-1-yl)-2H-chromen-2-ones as free radical scavengers. Eur. J. Med. Chem. 2011, 46, 5894-5901. [CrossRef]

8. Bernotas, R.C.; Singhaus, R.R.; Kaufman, D.H.; Ullrich, J.; Fletcher, H.; Quinet, E.; Nambi, P.; Unwalla, R.; Wilhelmsson, A.; Nilsson, A.G.; et al. Biarylether amide quinolines as liver $\mathrm{X}$ receptor agonists. Bioorg. Med. Chem. 2009, 17, 1663-1670. [CrossRef]

9. Facchini, P.J. Alkaloid biosynthesis in plants: Biochemistry, cell biology, molecular regulation and metabolic engineering applications. Ann. Rev. Plant Biol. 2001, 52, 29-66. [CrossRef]

10. Okunade, A.L. Ageratum conyzoids L. (Asteraceae). Fitoterapia 2002, 73, 1-16. [CrossRef]

11. Cheenpracha, S.; Karalai, C.; Pronglimanont, C.; Kanjana-Opas, A. Candenatenins A-F, phenolic compounds from the heartwood of Dalbergia candenatensis. J. Nat. Prod. 2009, 72, 1395-1398. [CrossRef] [PubMed]

12. Chung, P.-Y.; Bian, Z.-X.; Pun, H.-Y.; Chan, D.; Chan, A.S.-C.; Chui, C.-H.; Tang, J.C.-O.; Lam, K.-H. Recent advances in research of natural and synthetic bioactive quinolines. Future Med. Chem. 2015, 7, 947-967. [CrossRef] [PubMed]

13. Li, R.; Chen, X.; Song, X.-R.; Ding, H.; Wang, P.; Xiao, Q.; Liang, Y.-M. Copper-catalyzed cascade cyclization of 2-propynolphenols: Access to 4-phosphorylated 2H-chromenes. Adv. Synth. Catal. 2017, 359, 3962-3967. [CrossRef] 
14. Bogza, Y.P.; Rastrepin, A.A.; Nider, V.V.; Zheleznova, T.Y.; Stasyuk, A.J.; Kurowska, A.; Laba, K.; Ulyankin, E.B.; Domagala, W.; Fisyuk, A.S. Synthesis and optical properties of 2-functionally substituted 4,5-dihydrothieno[3,2-c]quinolines. Dye Pigment 2018, 159, 419-428. [CrossRef]

15. Ren, M.; Wang, S.; Yang, C.; Xu, H.; Guo, Y.; Roekaerts, D. Supercritical water oxidation of quinoline with moderate preheat temperature and initial concentration. Fuel 2019, 236, 1408-1414. [CrossRef]

16. Rocha, D.H.A.; Vaz, P.A.A.M.; Pinto, D.C.G.A.; Silva, A.M.S. Synthesis of chalcones and their isomerization into flavanones and azaflavanones. Methods Protoc. 2019, 2, 70. [CrossRef]

17. Sandulache, A.; Silva, A.M.S.; Cavaleiro, J.A.S. Diels-Alder reactions of chromone-3-carboxaldehydes with ortho-benzoquinodimethane. New synthesis of benzo[b]xanthones. Tetrahedron 2002, 58, 105-114. [CrossRef]

18. Coelho, A.; El-Maatougui, A.; Raviña, E.; Cavaleiro, J.A.S.; Silva, A.M.S. Efficient consecutive alkylation-Knoevenagel functionalisations in formyl aza-heterocycles using supported organic bases. Synlett 2006, 2006, 3324-3328. [CrossRef]

19. Kochanowska-Karamyan, A.J.; Hamann, M.T. Marine indole alkaloids: Potential new drug leads for the control of depression and anxiety. Chem. Rev. 2010, 110, 4489-44971. [CrossRef]

20. Chadha, N.; Silakari, O. Indoles as therapeutics of interest in medicinal chemistry: Bird's eye view. Eur. J. Med. Chem. 2017, 134, 159-184. [CrossRef]

21. Abdelfatah, S.A.A.; Efferth, T. Cytotoxicity of the indole alkaloid reserpine from Rauwolfia serpentine against drug-resistant tumor cells. Phytomedicine 2015, 22, 308-318. [CrossRef] [PubMed]

22. Crump, M.; Leppä, S.; Fayad, L.; Lee, J.J.; Rocco, A.D.; Ogura, M.; Hagberg, H.; Schnell, F.; Rifkin, R.; Mackensen, A.; et al. Randomized, double-blind, Phase III trial of enzastaurin versus placebo in patients achieving remission after first-line therapy for high-risk diffuse large B-cell lymphoma. J. Clin. Oncol. 2016, 34, 2484-2492. [CrossRef] [PubMed]

23. Xia, Q.; Bao, X.; Sun, C.; Wu, D.; Rong, X.; Liu, Z.; Gu, Y.; Zhou, J.; Liang, G. Design, synthesis and biological evaluation of novel 2-sulfonylindoles as potential anti-inflammatory therapeutic agents for treatment of acute lung injury. Eur. J. Med. Chem. 2018, 160, 120-132. [CrossRef] [PubMed]

24. Hurdle, J.G.; O’Neill, A.J.; Chopra, I. Anti-staphylococcal activity of indolimycin, a potential topical agent for control of staphylococcal infections. J. Antimicrob. Chemother. 2004, 54, 549-552. [CrossRef] [PubMed]

25. Sanna, G.; Madeddu, S.; Giliberti, G.; Piras, S.; Struga, M.; Wrzosek, M.; Kubiak-Tomaszewska, G.; Koziol, A.E.; Savchenko, O.; Lis, T.; et al. Synthesis and biological evaluation of novel indole-derived thioureas. Molecules 2018, 23, 2554. [CrossRef]

26. Wu, S.; Yang, Q.; Hu, Q.; Wang, Y.; Chen, L.; Zhang, H.; Wu, L.; Li, J. Manganese-catalyzed direct C2-allylation of indoles. Org. Chem. Front. 2018, 5, 2852-2855. [CrossRef]

27. Huang, Z.; Kwon, O.; Huang, H.; Fadli, A.; Marat, X.; Moreau, M.; Lumb, J.-P. A bioinspired synthesis of polyfunctional indoles. Angew. Chem. Int. Ed. 2018, 57, 11963-11967. [CrossRef]

28. Uesawa, Y.; Sakagami, H.; Ishihara, M.; Kagaya, H.; Kanamoto, T.; Terakubo, S.; Nakashima, H.; Yahagi, H.; Takao, K.; Sugita, Y. Quantitative structure-cytotoxicity relationship of 3-styryl-2H-chromenes. Anticancer Res. 2015, 35, 5299-5308.

29. Panda, P.; Nayak, S.; Bhakta, S.; Mohapatra, S.; Murthy, T.R. Design and synthesis of (Z/E)-2-phenyl/H-3styryl-2H-chromene derivatives as antimicrotubule agents. J. Chem. Sci. 2018, 130, 127. [CrossRef]

30. Sandulache, A.; Silva, A.M.S.; Pinto, D.C.G.A.; Almeida, L.M.P.M.; Cavaleiro, J.A.S. Wittig reactions of chromone-3-carboxaldehydes with benzylidenetriphenyl phosphoranes: A new synthesis of 3-styrylchromones. New J. Chem. 2003, 27, 1592-1598. [CrossRef]

31. Robiette, R.; Richardson, J.; Aggarwal, V.; Harvey, J. Reactivity and selectivity in the Wittig reaction: A computational study. J. Am. Chem. Soc. 2006, 128, 2394-2409. [CrossRef] [PubMed]

32. Seixas, R.S.G.R.; Silva, A.M.S.; Cavaleiro, J.A.S. New synthesis of (Z)-and (E)-3-styryl-4-quinolones. Synlett 2010, 2010, 2257-2262. [CrossRef]

33. Frisch, M.J.; Trucks, G.W.; Schlegel, H.B.; Scuseria, G.E.; Robb, M.A.; Cheeseman, J.R.; Scalmani, G.; Barone, V.; Mennucci, B.; Petersson, G.A.; et al. Gaussian 09, Revision, A.2; Gaussian: Wallingford, UK, 2009.

34. Becke, A.D. Density-functional thermochemistry. III. The role of exact exchange. J. Chem. Phys. 1993, 98, 5648-5652. [CrossRef] 
35. Lee, C.T.; Yang, W.T.; Parr, R.G. Development of the Colle-Solvetti correlation-energy formula into a functional of the electron density. Phys. Rev. B 1988, 37, 785-789. [CrossRef] [PubMed]

36. Hariharan, P.C.; Pople, J.A. The influence of polarization functions on molecular orbital hydrogenation energies. Theor. Chim. Acta 1973, 28, 213-222. [CrossRef]

Sample Availability: Samples of the compounds are not available from the authors.

(C) 2020 by the authors. Licensee MDPI, Basel, Switzerland. This article is an open access article distributed under the terms and conditions of the Creative Commons Attribution (CC BY) license (http://creativecommons.org/licenses/by/4.0/). 